\title{
Numerical and analytic method for solvingproposal New Type for fuzzy nonlinear volterra integral equation
}

\author{
Sameer Qasim Hasan \\ Department of Mathematics, College of Education, University of Al-Mustansiriyah, Baghdad Iraq \\ dr.sameer_Kkasim@yahoo.com \\ Alan jalal Abdulqader \\ Department of Mathematics, College of Education, University of Al-Mustansiriyah, Baghdad Iraq \\ alanjalal515@yahoo.com
}

\begin{abstract}
In this paper, we proved the existence and uniqueness and convergence of the solution of new type for nonlinear fuzzy volterra integral equation. The homotopy analysis method are proposed to solve the new type fuzzy nonlinear Volterra integral equation. We convert a fuzzy volterra integral equation for new type of kernel for integral equation, to a system of crisp function nonlinear volterra integral equation. We use the homotopy analysis method to find the approximate solution of the system and hence obtain an approximation for fuzzy solution of the nonlinear fuzzy volterra integral equation. Some numerical examples is given and results reveal that homotopy analysis method is very effective and compared with the exact solution and calculate the absolute error between the exact and AHM .Finally using the MAPLE program to solve our problem .
\end{abstract}

Keywords: Fuzzy Number; Volterra nonlinear Integral equation; fuzzy integral; Homotopy analysis method

\section{Introduction}

The solutions of integral equations have a major role in the field of science and engineering. Since few of these equations can be solved explicitly, it is often necessary to resort to numerical techniques which are appropriate combinations of numerical integration and interpolation [7, 12]. There are several numerical methods for solving linear Volterra integral equation [11, 23] and system of nonlinear Volterra integral equations [3]. Borzabadi and Fard in [5] obtained a numerical solution of nonlinear Fredholm integral equations of the second kind.The concept of fuzzy numbers and fuzzy arithmetic operations were first introduced by Zadeh [14],Dubois and Prade [21]. We refer the reader to [10] for more information on fuzzy numbers and fuzzy arithmetic. The topics of fuzzy integral [20] and fuzzy integral equations (FIE) which growing interest for some time, inparticular in relation to fuzzy control, have been rapidly developed in recent years. The fuzzy mapping functionwas introduced by Chang and Zadeh [14]. Later, Dubois and Prade [8] presented an elementary fuzzy calculusbased on the exten- sion principle also the concept of integration of fuzzy functions was first introduced byDubois and Prade [21]. Babolian et al. and Abbasbandy et al. in [10,11] obtained a numerical solution of linearFredholm fuzzy integral equations of the second kind, while finding an approximate solution for the fuzzynonlinear kinds.is more difficult and a numerical method in this case can be found in [4]In this paper, we present a novel and very simply numerical method ( Homotopy Analysis method ) for solving fuzzy nonlinear volterra integral equation .

.2.Basic concepts Basic definitions of fuzzy number are given in $[1,2,10,15,17,20]$ as follows:

Definition 2.1. Fuzzy number. A fuzzy number is a map $u: R \rightarrow[a, b]$, which satisfying

(1) $u$ is upper semi- continuous function,

(2) $u(x)=0$ outside some interval $[a, d]$

(3) There are real numbers b,c such $\mathrm{a} \leq \mathrm{b} \leq \mathrm{c} \leq \mathrm{d}$

i) $\mathrm{u}(\mathrm{x})$ is a monotonic increasing function on $[\mathrm{a}, \mathrm{b}]$

ii) $u(x)$ is a monotonic decreasing function on $[c, d]$

iii) $u(x)=1 \quad$ for all $x \in[b, c]$

The set of all fuzzy numbers (as given by Definition 13 ) is denoted by $\mathrm{E}^{1}$ and is a convex cone. An alternative definition for parameter from of a fuzzy number is given by Kaleva [14 ].

Definition 2.2. A fuzzy number $\breve{u}$ in parametric form is a pair $(\underline{u}, \bar{u})$ of function $\underline{u}(\alpha), \bar{u}(\alpha), \quad 0 \leq \alpha \leq 1$, which satisfies the following requiremenst:

i) $\underline{u}(\alpha)$ is a bounded left continuous non- decreasing function over [0, 1$]$

ii) $\overline{\mathrm{u}}(\alpha)$ is a bounded left continuous non- increasing function over $[0,1]$

iii) $\underline{\mathrm{u}}(\alpha) \leq \overline{\mathrm{u}}(\alpha), 0 \leq \alpha \leq 1$ 
Definition 2.3. . For arbitrary fuzzy $\mathrm{u}=(\underline{\mathrm{u}}(\alpha), \overline{\mathrm{u}}(\alpha)), \mathrm{v}=(\underline{\mathrm{v}}(\alpha), \bar{v}(\alpha)),, 0 \leq \alpha \leq 1$ and scalar $\mathrm{k}$, we define addition, subtraction, scalar product by $\mathrm{k}$ and multiplication are respectively as following:

$1-$ addition : $(\underline{u+v)}(\alpha)=(\underline{u}(\alpha)+\underline{v}(\alpha)), \quad \overline{(\bar{u}+v)}(\alpha)=(\bar{u}(\alpha)+\bar{v}(\alpha))$,

$2-$ subtraction $:(\underline{\mathrm{u}-\mathrm{v})}(\alpha)=(\underline{\mathrm{u}}(\alpha) \underline{\mathrm{v}}(\alpha)), \quad \overline{(\overline{\mathrm{u}-\mathrm{v})}}(\alpha)=(\overline{\mathrm{u}}(\alpha)-\overline{\mathrm{v}}(\alpha))$,

3 - scalar product :

$$
k \breve{u}= \begin{cases}(k \underline{u}(\alpha), k \bar{u}(\alpha)), & k \geq 0 \\ (k \underline{u}(\alpha), k \bar{u}(\alpha)), & k<0\end{cases}
$$

Defined 2.4. For arbitrary Fuzzy numbers $\tilde{u}, \tilde{v} \in E^{1}$

$$
\mathrm{D}(\tilde{\mathrm{u}}, \tilde{\mathrm{v}})=\max \left[\operatorname{sip}_{\alpha \in[0,1]}|\underline{\mathrm{u}}(\alpha)-\underline{\mathrm{v}(\alpha)}| \operatorname{sip}_{\alpha \in[0,1]}[\overline{\mathrm{u}}(\alpha)-\overline{\mathrm{v}}(\alpha) \mid\}\right.
$$

In the distance between The $\widetilde{u}$ and $\tilde{v}$, it is prove $\left(\mathrm{E}^{1}, \mathrm{D}\right)$ is a complete metric space .

Definition 2.5. The integral of a fuzzy function was define in [14] by using the Riemann integral concept . Let $\mathrm{f}:[\mathrm{a}, \mathrm{b}] \rightarrow \mathrm{E}^{1}$. For Fuzzy function, for each partition $\mathrm{p}=\left\{\mathrm{t}_{0}, \ldots, \mathrm{t}_{\mathrm{n}}\right\}$ of $[\mathrm{a}, \mathrm{b}]$ and for arbitrary $\xi_{\mathrm{i}} \in\left[\mathrm{t}_{\mathrm{i}-1}, \mathrm{t}_{\mathrm{i}}\right] \quad, 1 \leq \mathrm{i} \leq \mathrm{n}$, suppose

$R_{P}=\sum_{i=1}^{n} f\left(\xi_{i}\right)\left(t_{i}-t_{i-1}\right)$

$$
\Delta:=\max \left\{\left|\mathrm{t}_{\mathrm{i}}-\mathrm{t}_{\mathrm{i}-1}\right|, 1 \leq \mathrm{i} \leq \mathrm{n}\right\}
$$

The define integral of $f(t)$ over $[a, b]$ is

$$
\int_{a}^{b} f(t) d t=\lim _{\Delta \rightarrow 0} R_{P},
$$

If the fuzzy function $f(t)$ is continuous in metric $D$,its definite the integral exists and also

$\left(\int_{\mathrm{a}}^{\mathrm{b}} \underline{\mathrm{f}(\mathrm{t} ; \alpha)} \mathrm{dt}\right)=\int_{\mathrm{a}}^{\mathrm{b}} \underline{\mathrm{f}}(\mathrm{t} ; \alpha) d \mathrm{t} \quad, \quad\left(\int_{\mathrm{a}}^{\mathrm{b}} \overline{\mathrm{f}(\mathrm{t} ; \alpha)} \mathrm{dt}\right)=\int_{\mathrm{a}}^{\mathrm{b}} \overline{\mathrm{f}}(\mathrm{t} ; \alpha) d \mathrm{t}$

It should be noted that the fuzzy integral can be also defined using the Lebesgue - type approach. However, if $f(t)$ is continuous, both approaches yield the same value. More details about the properties of the fuzzy integral

Proposition 2.1. A function $F, G: I \rightarrow E^{n}$ be integrable and $\varphi \in R . \in$ then

$$
\begin{aligned}
& \text { 1- } \int(F+G)=\int F+\int G \\
& \text { 2- } \int \varphi F=\varphi \int F \\
& \text { 3- } D(F, G) \text { IS integrable }
\end{aligned}
$$

$$
4-\mathrm{D}\left(\int \mathrm{F}, \int \mathrm{G}\right) \leq \int \mathrm{D}(\mathrm{F}, \mathrm{G})
$$

Proposition 2.2. For any $\mathrm{p}, \mathrm{q}, \mathrm{r}, \mathrm{s} \in \mathrm{E}^{\mathrm{n}}$ and $\varphi \in \mathrm{R}$, then the following hold

$\mathrm{i}-\left(\mathrm{E}^{\mathrm{n}}, \mathrm{D}\right)$ is a complete metric space

$\mathrm{ii}-\mathrm{D}(\varphi \mathrm{p}, \varphi \mathrm{q})=|\varphi| \mathrm{D}(\mathrm{p}, \mathrm{q})$

iii-D $(p+r, q+s)=D(p, q)$

$\operatorname{iv}-D(p+q, r+s) \leq D(p, r)+D(q, s)$

Definition2.6. A function $\mathrm{F}: \mathrm{I} \rightarrow \mathrm{E}^{\mathrm{n}}$ is called bounded if there exists a constant $\mathrm{M}>0$ such that $\mathrm{D}(\mathrm{F}(\mathrm{x}), \tilde{0}) \leq \mathrm{M}$ for all $\mathrm{x} \in$

Definition 2.7. A function $\mathrm{F}: \mathrm{I} \rightarrow \mathrm{E}^{\mathrm{n}}$ is said to be continuous if for arbitrary fixed $\mathrm{x}_{0} \in \mathrm{I}$ and $\varepsilon>0$ there exists $\delta>$ 0 such that if $\left|\mathrm{x}-\mathrm{x}_{0}\right|<\delta$, than $\mathrm{D}\left(\mathrm{F}(\mathrm{x}), \mathrm{F}\left(\mathrm{x}_{0}\right)\right)<\varepsilon$ for each $\mathrm{x} \in \mathrm{I}$

\section{Novel formula fuzzy nonlinear volterra integral equation}

The fuzzy nonlinear integral equation with integral kernel which is discussed in this work is the fuzzy nonlinear Volterra integral equation of the second kind (FNVIE-2) as follows:

$$
\tilde{\mathrm{u}}(\mathrm{x})=\tilde{\mathrm{f}}(\mathrm{x})+\lambda \int_{\mathrm{a}}^{\mathrm{x}} \mathrm{k}\left(\left(\mathrm{x}, \mathrm{t}, \tilde{\mathrm{F}}_{1}(\mathrm{x}, \mathrm{t}, \tilde{\mathrm{u}}(\mathrm{t})), \mathrm{G}\left(\mathrm{t}, \int_{\mathrm{a}}^{\mathrm{t}} \tilde{\mathrm{F}}_{2}(\mathrm{t}, \mathrm{s}, \tilde{\mathrm{u}}(\mathrm{s})) \mathrm{ds}\right)\right) \mathrm{dt}\right.
$$


where $\lambda \geq 0, \tilde{\mathrm{f}}(\mathrm{x})$ is a fuzzy function of $\mathrm{x}: \mathrm{a} \leq \mathrm{x} \leq \mathrm{b}, \mathrm{k}\left(\left(\mathrm{x}, \mathrm{t}, \widetilde{\mathrm{F}}_{1}(\mathrm{x}, \mathrm{t}, \tilde{\mathrm{u}}(\mathrm{t}))\right), \widetilde{\mathrm{G}}\left(\mathrm{t}, \int_{\mathrm{a}}^{\mathrm{t}} \widetilde{\mathrm{F}}_{2}(\mathrm{t}, \mathrm{s}, \tilde{\mathrm{u}}(\mathrm{s})) \mathrm{ds}\right)\right)$ isanalytic functionson $[\mathrm{a}, \mathrm{b}] \times$ $[a, b] \times[a, b] \times E^{n} \times E^{n}$ and $\tilde{F}_{1}(x, t, \tilde{u}(t)), \tilde{F}_{2}(t, s, \tilde{u}(s))$ are nonlinear function on $[a, b]$. For solving in parametric form of Eq. (6), consider $(\underline{f}(x, \alpha), \bar{f}(x, \alpha))$ and $(\underline{u}(x, \alpha), \overline{\mathrm{u}}(x, \alpha)), 0 \leq \alpha \leq 1$ and $t, s \in[a, b]$ are parametric form of $\tilde{\mathrm{f}}(\mathrm{x})$ and $\tilde{u}(\mathrm{x})$, respectively. then, parametric form of Eq. (6) is as follows

$$
\begin{aligned}
& \left.\left.\underline{\mathrm{u}}(\mathrm{x}, \alpha)=\underline{\mathrm{f}}(\mathrm{x}, \alpha)+\lambda \underline{\int_{\mathrm{a}}^{\mathrm{x}} \mathrm{k}\left(\left(\mathrm{x}, \mathrm{t}, \mathrm{F}_{1 \alpha}(\mathrm{x}, \mathrm{t}, \mathrm{u}(\mathrm{t}, \alpha)), \mathrm{G}\left(\mathrm{t}, \int_{\mathrm{a}}^{\mathrm{t}} \mathrm{F}_{2 \alpha}(\mathrm{t}, \mathrm{s}, \mathrm{u}(\mathrm{s}, \alpha))\right.\right.\right.} \mathrm{ds}\right)\right) \mathrm{dt} \\
& \left.\left.\bar{u}(x, \alpha)=\bar{f}(x, \alpha)+\lambda \overline{\int_{a}^{x} k\left(\left(x, t, F_{1 \alpha}(x, t, u(t, \alpha)), G\left(t, \int_{a}^{t} F_{2 \alpha}(t, s, u(s, \alpha))\right.\right.\right.} d s\right)\right) d t
\end{aligned}
$$

let $x, t, s \in[a, b]$

$$
\begin{aligned}
& \frac{\mathrm{k}\left(\left(\mathrm{x}, \mathrm{t}, \mathrm{F}_{1 \alpha}(\mathrm{x}, \mathrm{t}, \mathrm{u}(\mathrm{t}, \alpha)), \mathrm{G}\left(\mathrm{t}, \int_{\mathrm{a}}^{\mathrm{t}} \mathrm{F}_{2 \alpha}(\mathrm{t}, \mathrm{s}, \mathrm{u}(\mathrm{s}, \alpha)) \mathrm{ds}\right)\right.\right.}{\mathrm{k}\left(\left(\mathrm{x}, \mathrm{t}, \mathrm{F}_{1 \alpha}(\mathrm{x}, \mathrm{t}, \mathrm{u}(\mathrm{t}, \alpha)), \mathrm{G}\left(\mathrm{t}, \int_{\mathrm{a}}^{\mathrm{t}} \mathrm{F}_{2 \alpha}(\mathrm{t}, \mathrm{s}, \mathrm{u}(\mathrm{s}, \alpha))\right.\right.\right.}=\left\{\begin{array}{c}
\mathrm{k}\left(\mathrm{x}, \mathrm{t}, \mathrm{F}_{1 \alpha}(\mathrm{t}, \underline{\mathrm{u}}(\mathrm{t}, \alpha)), \mathrm{k}(\mathrm{x}, \mathrm{t}) \geq 0\right. \\
\mathrm{k}\left(\mathrm{x}, \mathrm{t}, \mathrm{G}\left(\mathrm{t}, \int_{\mathrm{a}}^{\mathrm{t}} \mathrm{F}_{2 \alpha}(\mathrm{t}, \mathrm{s}, \overline{\mathrm{u}}(\mathrm{s}, \alpha)) \mathrm{ds}\right)\right), \mathrm{k}(\mathrm{x}, \mathrm{t})<0
\end{array}\right. \\
& \begin{array}{c}
\mathrm{k}\left(\mathrm{x}, \mathrm{t}, \mathrm{G}\left(\mathrm{t}, \int_{\mathrm{a}}^{\mathrm{t}} \overline{\mathrm{F}}_{2 \alpha}(\mathrm{t}, \mathrm{s}, \overline{\mathrm{u}}(\mathrm{s}, \alpha)) \mathrm{ds}\right)\right), \mathrm{k}(\mathrm{x}, \mathrm{t}) \geq 0 \\
\mathrm{k}\left(\mathrm{x}, \mathrm{t}, \mathrm{F}_{1 \alpha}(\mathrm{t}, \underline{\mathrm{u}}(\mathrm{t}, \alpha))\right), \mathrm{k}(\mathrm{x}, \mathrm{t})<0
\end{array}
\end{aligned}
$$

where

$$
\begin{aligned}
& \frac{\mathrm{k}\left(\mathrm{x}, \mathrm{t}, \mathrm{F}_{1 \alpha}(\mathrm{t}, \mathrm{u}(\mathrm{t}, \alpha))\right.}{\alpha}=\mathrm{k}\left(\mathrm{x}, \mathrm{t}, \underline{\mathrm{F}_{1 \alpha}}(\mathrm{t}, \mathrm{u}(\mathrm{t}, \mathrm{\alpha}))\right)=\mathrm{k}\left(\mathrm{x}, \mathrm{t}, \mathrm{F}_{1 \alpha}(\mathrm{t}, \underline{\mathrm{u}}(\mathrm{t}, \mathrm{\alpha}))\right) \text { and } \\
& \overline{\mathrm{k}\left(\mathrm{x}, \mathrm{t}, \mathrm{F}_{1 \alpha}(\mathrm{t}, \mathrm{u}(\mathrm{t}, \mathrm{\alpha}))\right.} \mathrm{k}\left(\mathrm{x}, \mathrm{t}, \overline{\mathrm{F}_{1 \alpha}}(\mathrm{t}, \mathrm{u}(\mathrm{t}, \alpha))\right)=\mathrm{k}\left(\mathrm{x}, \mathrm{t}, \mathrm{F}_{1 \alpha}(\mathrm{t}, \overline{\mathrm{u}}(\mathrm{t}, \alpha))\right)
\end{aligned}
$$

For each $0 \leq a \leq 1$ anda $\leq x \leq b$. WecanseethatEq. (6)converttoasystem of nonlinear Volterra integral equations in crisp case for each $0 \leq \alpha \leq 1$ and $a \leq t \leq b$. Now, we explain homotopy analysis methods as approximating solution of this system of nonlinear integral equations in crisp case. then, we find approximate solutions for $\tilde{u}(x), a \leq x \leq b \quad 0 \leq c \leq x$

\subsection{Homotopy analytic method "HAM"}

Now we apply homotopy analytic method for solve the system (7) and obtain a recursion scheme for it. Prior to apply HAM for the system (7). We suppose that the kernel have four cases for kernal's .

$$
\begin{gathered}
\mathrm{N}[\tilde{\mathrm{u}}(\mathrm{x}, \alpha)]=0 \\
\tilde{\mathrm{u}}(\mathrm{x})=\tilde{\mathrm{f}}(\mathrm{x})+\lambda \int_{\mathrm{a}}^{\mathrm{x}} \mathrm{k}\left(\left(\mathrm{x}, \mathrm{t}, \widetilde{\mathrm{F}}_{1}(\mathrm{x}, \mathrm{t}, \tilde{\mathrm{u}}(\mathrm{t})), \mathrm{G}\left(\mathrm{t}, \int_{\mathrm{a}}^{\mathrm{t}} \tilde{\mathrm{F}}_{2}(\mathrm{t}, \mathrm{s}, \tilde{\mathrm{u}}(\mathrm{s})) \mathrm{ds}\right)\right) \mathrm{dt}\right.
\end{gathered}
$$

Where

$\tilde{\mathrm{u}}(\mathrm{x}, \alpha)=(\underline{\mathrm{u}}(\mathrm{x}, \alpha), \overline{\mathrm{u}}(\mathrm{x}, \alpha))$ and $\tilde{\mathrm{f}}(\mathrm{x}, \alpha)=(\underline{\mathrm{f}}(\mathrm{x}, \alpha), \overline{\mathrm{f}}(\mathrm{x}, \alpha))$

We see that eq(15) is convert to system of nonlinear crisp fuzzy volterra integral equations

$$
\begin{array}{r}
\underline{\mathrm{u}}(\mathrm{x}, \alpha)=\underline{\mathrm{f}}(\mathrm{x}, \alpha)+\lambda \int_{\mathrm{a}}^{\mathrm{c}} \mathrm{k}\left(\mathrm{x}, \mathrm{t}, \underline{\mathrm{F}}_{1 \alpha}(\mathrm{t}, \underline{\mathrm{u}}(\mathrm{t}, \alpha))\right) \mathrm{dt}+\lambda \int_{\mathrm{c}}^{\mathrm{x}} \mathrm{k}\left(\mathrm{x}, \mathrm{t}, \mathrm{G}\left(\mathrm{t}, \int_{\mathrm{a}}^{\mathrm{t}} \overline{\mathrm{F}}_{2 \alpha}(\mathrm{t}, \mathrm{s}, \overline{\mathrm{u}}(\mathrm{s}, \alpha)) \mathrm{ds}\right) \mathrm{dt}\right) \\
\overline{\mathrm{u}}(\mathrm{x}, \alpha)=\overline{\mathrm{f}}(\mathrm{x}, \alpha)+\lambda \int_{\mathrm{a}}^{\mathrm{c}} \mathrm{k}\left(\mathrm{x}, \mathrm{t}, \mathrm{G}\left(\mathrm{t}, \int_{\mathrm{a}}^{\mathrm{t}} \overline{\mathrm{F}}_{2 \alpha}(\mathrm{t}, \mathrm{s}, \overline{\mathrm{u}}(\mathrm{s}, \alpha)) \mathrm{ds}\right) \mathrm{dt}\right)+\lambda \int_{\mathrm{c}}^{\mathrm{x}} \mathrm{k}\left(\mathrm{x}, \mathrm{t}, \underline{\mathrm{F}}_{1 \alpha}(\mathrm{t}, \underline{\mathrm{u}}(\mathrm{t}, \alpha))\right) \mathrm{dt}
\end{array}
$$

(9)

For solving system (9) by HAM, we construct the zeroth-order deformation equations:

$$
\begin{gathered}
(1-p) L\left[\underline{\emptyset}(x, p ; \alpha)-\underline{w}_{0}(x ; \alpha)\right]=\operatorname{phH}[\underline{\emptyset}(x, p ; \alpha)-\underline{f}(x, \alpha) \\
-\lambda\left[\int _ { a } ^ { c } k \left(\left(x, t, \underline{F}_{1}(x, t, \underline{\emptyset}(t, p ; \alpha))\right)+\int_{c}^{x} k\left(x, t, G\left(t, \int_{a}^{t} \bar{F}_{2 \alpha}(t, s, \bar{\emptyset}(s, p, \alpha)) d s\right) d t\right]\right.\right.
\end{gathered}
$$




$$
\begin{array}{r}
(1-p) L\left[\bar{\varnothing}(x, p ; \alpha)-\bar{w}_{0}(x ; \alpha)\right]=p h H[\bar{\varnothing}(x, p ; \alpha)-\bar{f}(x, \alpha) \\
-\lambda\left[\int _ { a } ^ { c } k \left(x, t, G\left(t, \int_{a}^{t} \bar{F}_{2 \alpha}(t, s, \bar{\varnothing}(s, p, \alpha)) d s\right) d t+\int_{c}^{x} k\left(\left(x, t, \underline{F}_{1 \alpha}(x, t, \underline{\phi}(t, p ; \alpha)) d t\right]\right.\right.\right.
\end{array}
$$

Where $p \in[a, b]$ is the embedding parameter, his nonzero auxiliary parameter, $L$ is an auxiliary linear operator $H(x)$ is an auxiliary function, $\left(\underline{w}_{0}(x ; \alpha), \bar{w}_{0}(x ; \alpha)\right)$ are initial guesses of $\underline{F}_{1 \alpha}(x, t, \underline{\emptyset}(t, p ; \alpha)), \bar{F}_{1 \alpha}(t, s, \bar{\emptyset}(t, p ; \alpha)), \bar{F}_{2 \alpha}(x, t, \bar{\emptyset}(t, p ; \alpha))$ and $\underline{F}_{2 \alpha}(t, s, \underline{\emptyset}(t, p ; \alpha))$ respectively and $\underline{\emptyset}(t, p ; \alpha)$ and $\bar{\emptyset}(t, p ; \alpha)$ are unknown functions. Using the above zeroth - order deformation equations, with assumption

$L(u)=u$ and $H(x)=1$, we have

$$
\begin{gathered}
(1-p)\left[\underline{\emptyset}(x, p ; \alpha)-\underline{w}_{0}(x ; \alpha)\right]=p h[\underline{\emptyset}(x, p ; \alpha)-\underline{f}(x, \alpha) \\
-\lambda\left[\int _ { a } ^ { c } k \left(\left(x, t, \underline{F}_{1 \alpha}(x, t, \underline{\emptyset}(t, p ; \alpha))\right)+\int_{c}^{x} k\left(x, t, G\left(t, \int_{a}^{t} \bar{F}_{2 \alpha}(t, s, \bar{\emptyset}(s, p, \alpha)) d s\right) d t\right]\right.\right. \\
(1-p)\left[\bar{\emptyset}(x, p ; \alpha)-\bar{w}_{0}(x ; \alpha)\right]=p h[\bar{\varnothing}(x, p ; \alpha)-\bar{f}(x, \alpha) \\
-\lambda\left[\int _ { a } ^ { c } k \left(x, t, G\left(t, \int_{a}^{t} \bar{F}_{2 \alpha}(t, s, \bar{\emptyset}(s, p, \alpha)) d s\right)+\int_{c}^{x} k\left(\left(x, t, \underline{F}_{1 \alpha}(x, t, \underline{\emptyset}(t, p ; \alpha))\right) d t\right]\right.\right.
\end{gathered}
$$

Obviously, when $p=0$ and $p=1$ and since $h \neq 0$, we obtain

$$
\underline{\emptyset}(x, 0 ; \alpha)=\underline{w}_{0}(x ; \alpha)
$$

$\bar{\emptyset}(x, 0 ; \alpha)=\bar{w}_{0}(x ; \alpha)$

And

$$
\underline{\emptyset}(x, 1 ; \alpha)=\underline{f}(x, \alpha)+\lambda \int_{a}^{c} k\left(\left(x, t, \underline{F}_{1 \alpha}(x, t, \underline{\emptyset}(t, 1 ; \alpha))\right) d t+\lambda \int_{c}^{x} k\left(x, t, G\left(t, \int_{a}^{t} \bar{F}_{2 \alpha}(t, s, \bar{\emptyset}(s, 1, \alpha)) d s\right) d t\right.\right.
$$

$\bar{\emptyset}(x, 1 ; \alpha)=\bar{f}(x, \alpha)+\lambda \int_{a}^{c} k\left(x, t, G\left(t, \int_{a}^{t} \bar{F}_{2 \alpha}(t, s, \bar{\emptyset}(s, 1, \alpha)) d s\right) d t+\int_{a}^{x} k\left(\left(x, t, \underline{F_{1 \alpha}}(x, t, \underline{\emptyset}(t, 1 ; \alpha))\right) d t(12)\right.\right.$

Respectively. Thus as $p$ increases from 0 to 1 , the function $\emptyset(x, p ; \alpha)$

$\bar{\emptyset}(x, p ; \alpha)$ deforms from the initial guesses $\underline{w}_{0}(x ; \alpha), \bar{w}_{0}(x ; \alpha)$ to the solution of $\underline{F}_{1 \alpha}(x, t, \underline{\emptyset}(t, p ; \alpha)), \bar{F}_{1 \alpha}(t, s, \bar{\emptyset}(t, p ; \alpha)), \bar{F}_{2 \alpha}(x, t, \bar{\emptyset}(t, p ; \alpha))$ and $\underline{F}_{2 \alpha}(t, s, \underline{\emptyset}(t, p ; \alpha))$.

Expanding $\underline{\emptyset}(x, p ; \alpha)$ in Taylors series with respect $p$ gives

$$
\begin{aligned}
\underline{\emptyset}(x, p ; \alpha)=\underline{w}_{0}(x ; \alpha)+\sum_{m=1}^{\infty} \underline{u}_{m}(x, \alpha) p^{m} \\
\bar{\emptyset}(x, p ; \alpha)=\bar{w}_{0}(x ; \alpha)+\sum_{m=1}^{\infty} \bar{u}_{m}(x, \alpha) p^{m}
\end{aligned}
$$

Where

$$
\underline{u}_{m}(x, r)=\left.\frac{1}{m !} \frac{\partial^{m} \emptyset(t, p ; r)}{\partial p^{m}}\right|_{p=0}
$$

$\bar{u}_{m}(x, r)=\left.\frac{1}{m !} \frac{\partial^{m} \bar{\emptyset}(t, p ; r)}{\partial p^{m}}\right|_{p=0} \quad, \quad m \geq 1$ 
It should be noted that $\underline{\emptyset}(x, p ; \alpha)=\underline{w}_{0}(x ; \alpha)$ and $\bar{\emptyset}(x, p ; \alpha)=\bar{w}_{0}(x ; \alpha)$ differential the zerothorder deformation equation (10) $m$ times with respect to the embedding parameter $p$ and dividing them by $m$ ! and $n$ ! finally setting $p=0$, we obtain the so called $m$-th order deformation equations

$$
\begin{aligned}
& \underline{u}_{m}(x ; r)=h\left[\underline{u}_{m-1}(x ; r)-\left(1-x_{m}\right) \underline{f}(x ; r)-\lambda \int_{a}^{c} \underline{\boldsymbol{R}}_{m-1}(x, t ; \alpha) d t-\lambda \int_{c}^{x} \overline{\boldsymbol{R}}_{m-1}(x, t ; \alpha) d t\right. \\
& \bar{u}_{m}(x ; r)=h\left[\bar{u}_{m-1}(x ; r)-\left(1-x_{m}\right) \bar{f}(x ; r)-\lambda \int_{c}^{x} \overline{\boldsymbol{R}}_{m-1}(x, t ; \alpha) d t-\lambda \int_{a}^{c} \underline{\boldsymbol{R}}_{m-1}(x, t ; \alpha) d t\right.
\end{aligned}
$$

Where

$$
\begin{gathered}
x_{m}=\left\{\begin{array}{cc}
0, m=1 \\
1, m \neq 1
\end{array} \quad m \geq 1\right. \\
\underline{\emptyset}(x, \alpha)=\underline{w}_{0}(x ; \alpha), \text { and } \\
\underline{\boldsymbol{R}}_{m-1}(x, t ; \alpha)=\left.\frac{1}{(m-1) !} \frac{\partial^{m-1} k\left(x, t, \underline{F}_{1}(x, \underline{\emptyset}(x, p ; \alpha))\right.}{\partial p^{m-1}}\right|_{p=0}
\end{gathered}
$$

and

$\bar{\emptyset}(x, \alpha)=\bar{w}_{0}(x ; \alpha)$

$\overline{\boldsymbol{R}}_{m-1}(x, t ; \alpha)=\left.\frac{1}{(m-1) !} \frac{\partial^{m-1} k\left(x, t, G\left(t, \int_{a}^{t} \bar{F}_{2 \alpha}(t, s, \bar{\emptyset}(s, p, \alpha) d s)\right.\right.}{\partial p^{m-1}}\right|_{p=0}$

From Eqs (14) and (15), we have that

$$
\begin{gathered}
\underline{u}_{0}(x, \alpha)=0 \\
\underline{u}_{1}(x ; \alpha)=h \underline{u}_{0}(x ; \alpha)-h \underline{f}(x ; \alpha)-\lambda h\left[\int_{0}^{c} \underline{\boldsymbol{R}}_{0}(x, t ; \alpha) d t+\int_{c}^{x} \overline{\boldsymbol{R}}_{0}(x, t ; \alpha) d t\right] \\
\underline{u}_{m}(x ; \alpha)=(1+h) \underline{u}_{m-1}(x ; \alpha)-\lambda h\left[\int_{0}^{c} \underline{\boldsymbol{R}}_{m-1}(x, t ; \alpha) d t+\int_{c}^{x} \overline{\boldsymbol{R}}_{m-1}(x, t ; \alpha) d t\right]
\end{gathered}
$$

Similary

$$
\begin{gathered}
\bar{u}_{0}(x, \alpha)=0 \\
\bar{u}_{1}(x ; \alpha)=h \underline{u}_{0}(x ; \alpha)-h \underline{f}(x ; \alpha)-\lambda h\left[\int_{0}^{c} \overline{\boldsymbol{R}}_{0}(x, t ; \alpha) d t+\int_{c}^{x} \underline{\boldsymbol{R}}_{0}(x, t ; \alpha) d t\right] \\
\bar{u}_{m}(x ; \alpha)=(1+h) \underline{u}_{m-1}(x ; \alpha)-\lambda h\left[\int_{0}^{c} \overline{\boldsymbol{R}}_{m-1}(x, t ; \alpha) d t+\int_{c}^{x} \underline{\boldsymbol{R}}_{m-1}(x, t ; \alpha) d t\right]
\end{gathered}
$$

where,

$$
\begin{gathered}
\underline{\boldsymbol{R}}_{0}(x, t ; \alpha)=k\left(x, t, \underline{F_{1 \alpha}}\left(t, \underline{u_{0}}(t, \alpha)\right)\right. \\
\overline{\boldsymbol{R}}_{0}(x, t ; \alpha)=k\left(x, t, G\left(t, \int_{a}^{t} \bar{F}_{2 \alpha}\left(t, s, \bar{u}_{0}(s, \alpha)\right) d s\right)\right.
\end{gathered}
$$

Thus, if we choose a proper values of $h$, the series (8) is convergent at $p=1$. Hence the solution of system (9) in series from (homotopy solution series ) is obtain as 


$$
\underline{U}(x, \alpha)=\underline{u}_{0}(x ; \alpha)+\sum_{m=1}^{\infty} \underline{u}_{m}(x ; \alpha)
$$

$\bar{U}(x, \alpha)=\bar{u}_{0}(x ; \alpha)+\sum_{m=1}^{\infty} \bar{u}_{m}(x ; \alpha)$

We denoted the $n-t h$ order approximate solution with

$$
\underline{U_{j}}(x, r)=\underline{u}_{0}(x ; r)+\sum_{m=1}^{\infty} \underline{u}_{m}(x ; r)
$$

$\overline{U_{j}}(x, r)=\bar{u}_{0}(x ; r)+\sum_{m=1}^{\infty} \bar{u}_{m}(x ; r)$

$$
j \geq 1
$$

Theorem 2.1: (. Existence and uniqueness)

The following conditions are satisfy

1- $f:[a, b] \rightarrow E^{n}$ is a continuous and bounded

2- $K:[a, b] \times[a, b] \times[a, b] \times E^{n} \times E^{n} \rightarrow E^{n}$ is a continuous function

3- if $u, u^{*}:[a, b] \rightarrow E^{n}$ are continuous, then the lipschitz condition

$$
\begin{array}{r}
D\left(k \left(x_{1}, t_{1}, \widetilde{H}_{11}(t), G\left(t, H_{12}(t), k\left(x_{2}, t_{2}, \widetilde{H}_{21}(t), G\left(t, H_{22}(t)\right) \leq L\left(D\left(x_{1}, x_{2}\right)+\right.\right.\right.\right.\right. \\
D\left(t_{1}, t_{2}\right)+D\left(H_{11}(t), H_{21}(t)\right)+D\left(G\left(t_{1}, H_{12}(t)\right), G\left(t_{2}, H_{22}(t)\right)\right)
\end{array}
$$

4- $D\left(F_{1}\left(x_{1}, t_{1}, \tilde{u}(t)\right), F_{1}\left(x_{2}, t_{2}, \tilde{u}^{*}(t)\right) \leq L_{1}\left(D\left(x_{1}, x_{2}\right)+D\left(t_{1}, t_{2}\right)+D\left(\tilde{u}(x), \tilde{u}^{*}(x)\right)\right)\right.$

5- $D\left(F_{2}\left(s_{1}, t_{1}, \tilde{u}(t)\right), F_{2}\left(s_{2}, t_{2}, \tilde{u}^{*}(t)\right) \leq L_{2}\left(\left(D\left(s_{1}, s_{2}\right)+D\left(t_{1}, t_{2}\right)+D\left(\tilde{u}(x), \tilde{u}^{*}(x)\right)\right.\right.\right.$

6- $D\left(G\left(t_{1}, \int_{a}^{t} F_{2}\left(s_{1}, t_{1}, \tilde{u}(s)\right) d s, G\left(t_{2}, \int_{a}^{t} F_{2}\left(s_{2}, t_{2}, \tilde{u}(s)\right) d s\right) \leq L_{3}\left(D\left(t_{1}, t_{2}\right)+\right.\right.\right.$

$$
(b-a) L_{2}\left(D\left(s_{1}, s_{2}\right)+D\left(t_{1}, t_{2}\right)+D\left(\tilde{u}(x), \tilde{u}^{*}(x)\right)\right)
$$

We take an initial guess $\bar{u}_{0}(x)=\bar{f}(x)$, an auxiliary operator $L \bar{u}=\bar{u}$, an nonzero auxiliary parameter $h=-1$ and auxiliary function $H(x)=1$, this is substituted in $\mathrm{Eq}(19)$ to give the recurrence relation .

Then there exist a unique fuzzy solution of $\mathrm{u}(\mathrm{x})$ in $\mathrm{Eq}(19)$ and the successive iteration

$$
\omega_{0}(x)=f(x)
$$

$$
\omega_{n+1}(x)=f(x)+\sum_{i=1}^{n+1} \int_{a}^{x} k\left(\left(x, t, \tilde{F}_{1}\left(x, t, \tilde{u}_{i-1}(t)\right), G\left(t, \int_{a}^{t} \tilde{F}_{2}\left(t, s, \tilde{u}_{i-1}(s)\right) d s\right)\right) d t \quad n \geq 0\right.
$$

and $\mathrm{u}(\mathrm{x})$ is convergent uniformly on $[\mathrm{a}, \mathrm{b}]$ where

$$
\begin{gathered}
u_{0}(x)=f(x) \\
u_{n}(x)=\int_{a}^{x} k\left(\left(x, t, \tilde{F}_{1}\left(x, t, \tilde{u}_{n-1}(t)\right), G\left(t, \int_{a}^{t} \tilde{F}_{2}\left(t, s, \tilde{u}_{n-1}(s)\right) d s\right)\right) d t, \quad n \geq 1\right.
\end{gathered}
$$


Lemma 2.1: Consider $f(x)$ is a bounded on [a,b] then we will prove the $u_{n}(x)$, is a bounded and $u_{n}(x)$ is countinuous, where $n \geq 0$

Proof: we will proved the part 1 from lemma $2.1 u_{0}(x)=f(x)$ is a bounded. Now we proved $u_{n-1}(x)$ is a bounded . and proposition (2.1), we have

$$
\begin{gathered}
D\left(\tilde{u}_{n}(x), \tilde{0}\right)=D\left(\int_{a}^{x} k\left(\left(x, t, \tilde{F}_{1}\left(x, t, \tilde{u}_{n-1}(t)\right), G\left(t, \int_{a}^{t} \tilde{F}_{2}\left(t, s, \tilde{u}_{n-1}(s)\right) d s\right)\right) d t, \tilde{0}\right)\right. \\
\leq \int_{a}^{x} D\left(k \left(\left(x, t, \tilde{F}_{1}\left(x, t, \tilde{u}_{n-1}(t)\right), G\left(t, \int_{a}^{t} \tilde{F}_{2}\left(t, s, \tilde{u}_{n-1}(s)\right) d s\right), \tilde{0}\right) d t\right.\right. \\
\leq(b-a) L\left(D(x, \tilde{0})+D(t, \tilde{0})+D\left(\tilde{F}_{1}\left(x, t, \tilde{u}_{n-1}(t)\right), \tilde{0}\right)+D\left(G\left(t, \int_{a}^{t} \tilde{F}_{2}\left(t, s, \tilde{u}_{n-1}(s)\right) d s\right), \tilde{0}\right)\right. \\
\leq(b-a) L\left(D(x, \tilde{0})+D(t, \tilde{0})+L_{1}\left[D(x, \tilde{0})+D(t, \tilde{0})+D\left(\left(\tilde{u}_{n-1}(t)\right), \tilde{0}\right)\right]\right. \\
\quad+L_{2}\left[D(t, \tilde{0})+\int_{0}^{t} L_{3}\left[D(t, \tilde{0})+D(s, \tilde{0})+D\left(\tilde{u}_{n-1}(s), \tilde{0}\right]\right.\right. \\
\leq L\left(D(x, \tilde{0})+D(t, \tilde{0})+L_{1}\left[D(x, \tilde{0})+D(t, \tilde{0})+D\left(\left(\tilde{u}_{n-1}(t)\right), \tilde{0}\right)\right]+L_{2}[D(t, \tilde{0})\right. \\
\quad+(b-a) L_{3}\left[D(t, \tilde{0})+D(s, \tilde{0})+D\left(\tilde{u}_{n-1}(s), \tilde{0}\right)\right] \\
\leq(b-a)\left(L+L_{1}\right)\left(D(x, \tilde{0})+(b-a)\left[L+L_{1}+L_{2}+(b-a) L_{3}\right] D(t, \tilde{0})\right. \\
\left.+(b-a) L_{3} D(s, \tilde{0})+(b-a)\left[(b-a)\left(L_{3}+L\right)\right) D\left(\left(\tilde{u}_{n-1}(t)\right), \tilde{0}\right)\right] \\
\leq(b-a)\left(L+L_{1}\right) \sup D(x, 0)+(b-a)\left[\left(L+L_{1}+L_{2}\right)+(b-a) L_{3}\right) \sup D(t, \tilde{0}) \\
\left.+(b-a) L_{3} \sup D(s, \tilde{0})+(b-a)\left[(b-a)\left(L_{3}+L\right) s u p D\left(\tilde{u}_{n-1}(t)\right), \tilde{0}\right)\right]
\end{gathered}
$$

$\tilde{u}_{n}(x)$ is a bounded, $\tilde{0}$ is the zero function

Now we will proof part 2 from lemma $2.1, \tilde{u}_{n}(x)$ is a continuous by using propostion (2.1)and (2.2), we have, $a \leq x \leq x_{1} \leq b$

$$
\begin{aligned}
\left(\tilde{u}_{n}(x), \tilde{u}_{n}\left(x_{1}\right)\right)= & D\left(\int _ { a } ^ { x } k \left(\left(x, t, \tilde{F}_{1}\left(x, t, \tilde{u}_{n-1}(t)\right), G\left(t, \int_{a}^{t} \tilde{F}_{2}\left(t, s, \tilde{u}_{n-1}(s)\right) d s\right)\right) d t\right.\right. \\
& , \int_{a}^{x_{1}} k\left(\left(x_{1}, t, \tilde{F}_{1}\left(x_{1}, t, \tilde{u}_{n-1}(t)\right), G\left(t, \int_{a}^{t} \tilde{F}_{2}\left(t, s, \tilde{u}_{n-1}(s)\right) d s\right)\right) d t\right)
\end{aligned}
$$$$
=D\left(\int _ { a } ^ { x } k \left(\left(x, t, \tilde{F}_{1}\left(x, t, \tilde{u}_{n-1}(t)\right), G\left(t, \int_{a}^{t} \tilde{F}_{2}\left(t, s, \tilde{u}_{n-1}(s)\right) d s\right)\right) d t\right.\right. \text {, }
$$$$
, \int_{a}^{x} k\left(\left(x_{1}, t, \tilde{F}_{1}\left(x_{1}, t, \tilde{u}_{n-1}(t)\right), G\left(t, \int_{a}^{t} \tilde{F}_{2}\left(t, s, \tilde{u}_{n-1}(s)\right) d s\right)\right) d t\right) \text {, }
$$$$
\left.+\int_{x}^{x_{1}} k\left(\left(x_{1}, t, \tilde{F}_{1}\left(x_{1}, t, \tilde{u}_{n-1}(t)\right), G\left(t, \int_{a}^{t} \tilde{F}_{2}\left(t, s, \tilde{u}_{n-1}(s)\right) d s\right)\right) d t\right)\right)
$$$$
\leq D\left(\int _ { a } ^ { x } k \left(\left(\left(x, t, \tilde{F}_{1}\left(x, t, \tilde{u}_{n-1}(t)\right), G\left(t, \int_{a}^{t} \tilde{F}_{2}\left(t, s, \tilde{u}_{n-1}(s)\right) d s\right)\right) d t, \int_{a}^{x} k\left(\left(x_{1}, t, \tilde{F}_{1}\left(x_{1}, t, \tilde{u}_{n-1}(t)\right), G\left(t, \int_{a}^{t} \tilde{F}_{2}\left(t, s, \tilde{u}_{n-1}(s)\right) d s\right)\right) d t\right)\right)\right.\right.
$$

$$
+D\left(\int_{x}^{x_{1}} k\left(\left(x_{1}, t, \tilde{F}_{1}\left(x_{1}, t, \tilde{u}_{n-1}(t)\right), G\left(t, \int_{a}^{t} \tilde{F}_{2}\left(t, s, \tilde{u}_{n-1}(s)\right) d s\right)\right) d t\right), \tilde{0}\right)
$$

$\leq \int_{a}^{x} D\left(k\left(\left(x, t, \tilde{F}_{1}\left(x, t, \tilde{u}_{n-1}(t)\right), G\left(t, \int_{a}^{t} \tilde{F}_{2}\left(t, s, \tilde{u}_{n-1}(s)\right) d s\right)\right), k\left(\left(x_{1}, t, \tilde{F}_{1}\left(x_{1}, t, \tilde{u}_{n-1}(t)\right), G\left(t, \int_{a}^{t} \tilde{F}_{2}\left(t, s, \tilde{u}_{n-1}(s)\right) d s\right)\right) d t\right)\right.\right.$ 


$$
\left.+\int_{x}^{x_{1}} D\left(k\left(\left(x_{1}, t, \tilde{F}_{1}\left(x_{1}, t, \tilde{u}_{n-1}(t)\right), G\left(t, \int_{a}^{t} \tilde{F}_{2}\left(t, s, \tilde{u}_{n-1}(s)\right) d s\right)\right) d t\right)\right), \tilde{0}\right) d t
$$

$\leq(b-a) \sup D\left(k\left(\left(x, t, \tilde{F}_{1}\left(x, t, \tilde{u}_{n-1}(t)\right), G\left(t, \int_{a}^{t} \tilde{F}_{2}\left(t, s, \tilde{u}_{n-1}(s)\right) d s\right)\right), k\left(\left(x_{1}, t, \tilde{F}_{1}\left(x_{1}, t, \tilde{u}_{n-1}(t)\right), G\left(t, \int_{a}^{t} \tilde{F}_{2}\left(t, s, \tilde{u}_{n-1}(s)\right) d s\right)\right)\right)\right.\right.$

$$
\begin{gathered}
+\left(x_{1}-x\right)\left[\left(L+L_{1}\right) \sup D(x, 0)+\left[\left(L+L_{1}+L_{2}\right)+(b-a) L_{3}\right) \sup D(t, \tilde{0})\right. \\
\left.+L_{3} \sup D(s, \tilde{0})+\left[(b-a)\left(L_{3}+L\right) \sup D\left(\tilde{u}_{n-1}(t)\right), \tilde{0}\right)\right]
\end{gathered}
$$

$\mathrm{K}$ is continuous, we have

$$
D\left(\tilde{u}_{n}(x), \tilde{u}_{n}\left(x_{1}\right)\right) \rightarrow 0 \text { as } x \rightarrow x_{1}
$$

$u_{n}(x)$ is continuous on $[\mathrm{a}, \mathrm{b}]$

proof

we will prove that $\omega_{n}(x), n \geq 0$ are bounded on $[\mathrm{a}, \mathrm{b}]$. Then $\omega_{0}(x)=f(x)$

is a bounded by the assumption that $\omega_{n-1}(x)$ is a bounded, from $\mathrm{Eq}(20)$ and propostion (2.1) we will get.

$$
\begin{gathered}
D\left(\omega_{n}(x), \tilde{0}\right)=D\left(f(x)+\sum_{i=1}^{n} \int_{a}^{x} k\left(\left(x, t, \tilde{F}_{1}\left(x, t, \tilde{u}_{i-1}(t)\right), G\left(t, \int_{a}^{t} \tilde{F}_{2}\left(t, s, \tilde{u}_{i-1}(s)\right) d s\right)\right) d t, \tilde{0}\right)\right. \\
=D\left(f(x)+\sum_{i=1}^{n-1} \int_{a}^{x} k\left(\left(x, t, \tilde{F}_{1}\left(x, t, \tilde{u}_{i-1}(t)\right), G\left(t, \int_{a}^{t} \tilde{F}_{2}\left(t, s, \tilde{u}_{i-1}(s)\right) d s\right)\right) d t\right.\right. \\
\quad+\int_{a}^{x} k\left(\left(x, t, \tilde{F}_{1}\left(x, t, \tilde{u}_{n-1}(t)\right), G\left(t, \int_{a}^{t} \tilde{F}_{2}\left(t, s, \tilde{u}_{n-1}(s)\right) d s\right)\right) d t, \tilde{0}\right) \\
=D\left(\omega_{n-1}(x)+\int_{a}^{x} k\left(\left(x, t, \tilde{F}_{1}\left(x, t, \tilde{u}_{n-1}(t)\right), G\left(t, \int_{a}^{t} \tilde{F}_{2}\left(t, s, \tilde{u}_{n-1}(s)\right) d s\right)\right) d t, \tilde{0}\right)\right. \\
\leq D\left(\omega_{n-1}(x), \tilde{0}\right)+D\left(\int_{a}^{x} k\left(\left(x, t, \tilde{F}_{1}\left(x, t, \tilde{u}_{n-1}(t)\right), G\left(t, \int_{a}^{t} \tilde{F}_{2}\left(t, s, \tilde{u}_{n-1}(s)\right) d s\right)\right) d t, \tilde{0}\right)\right. \\
=D\left(\omega_{n-1}(x), \tilde{0}\right)+D\left(\omega_{n}(x), \tilde{0}\right)
\end{gathered}
$$

From lemma (2.1) we have that $\omega_{n}(x)$ is a bounded . and $\left\{\omega_{n}(x)\right\}_{n=0}^{\infty}$ is a sequence of bounded functions on $[\mathrm{a}, \mathrm{b}]$

We will prove $\omega_{n}(x)$ are continuous on [a,b] , by using Lemma (2.1) and proposition (2.2) and(2.2) for $a \leq x \leq x_{1} \leq b$, we get

$$
\begin{gathered}
D\left(\omega_{n}(x), \omega_{n}\left(x_{1}\right)\right)=D\left(\left(f(x)+\sum_{i=1}^{n} \int_{a}^{x} k\left(\left(x, t, \tilde{F}_{1}\left(x, t, \tilde{u}_{i-1}(t)\right), G\left(t, \int_{a}^{t} \tilde{F}_{2}\left(t, s, \tilde{u}_{i-1}(s)\right) d s\right)\right) d t\right.\right.\right. \\
, f\left(x_{1}\right)+\sum_{i=1}^{n} \int_{a}^{x_{1}} k\left(\left(x_{1}, t, \tilde{F}_{1}\left(x_{1}, t, \tilde{u}_{i-1}(t)\right), G\left(t, \int_{a}^{t} \tilde{F}_{2}\left(t, s, \tilde{u}_{i-1}(s)\right) d s\right)\right) d t\right) \\
\leq D\left(f(x), f\left(x_{1}\right)\right)+D\left(\sum _ { i = 1 } ^ { n } \int _ { a } ^ { x } k \left(\left(x, t, \tilde{F}_{1}\left(x, t, \tilde{u}_{i-1}(t)\right), G\left(t, \int_{a}^{t} \tilde{F}_{2}\left(t, s, \tilde{u}_{i-1}(s)\right) d s\right)\right) d t\right.\right.
\end{gathered}
$$




$$
\begin{aligned}
& \sum_{i=1}^{n} \int_{a}^{x_{1}} k\left(\left(x_{1}, t, \tilde{F}_{1}\left(x_{1}, t, \tilde{u}_{i-1}(t)\right), G\left(t, \int_{a}^{t} \tilde{F}_{2}\left(t, s, \tilde{u}_{i-1}(s)\right) d s\right)\right) d t\right) \\
& =D\left(f(x), f\left(x_{1}\right)\right)+D\left(\sum _ { i = 1 } ^ { n } \int _ { a } ^ { x } k \left(\left(x, t, \tilde{F}_{1}\left(x, t, \tilde{u}_{i-1}(t)\right), G\left(t, \int_{a}^{t} \tilde{F}_{2}\left(t, s, \tilde{u}_{i-1}(s)\right) d s\right)\right) d t,\right.\right. \\
& \sum_{i=1}^{n} \int_{a}^{x} k\left(\left(x_{1}, t, \tilde{F}_{1}\left(x_{1}, t, \tilde{u}_{i-1}(t)\right), G\left(t, \int_{a}^{t} \tilde{F}_{2}\left(t, s, \tilde{u}_{i-1}(s)\right) d s\right)\right) d t\right)+ \\
& \left.\sum_{i=1}^{n} \int_{x}^{x_{1}} k\left(\left(x_{1}, t, \tilde{F}_{1}\left(x_{1}, t, \tilde{u}_{i-1}(t)\right), G\left(t, \int_{a}^{t} \tilde{F}_{2}\left(t, s, \tilde{u}_{i-1}(s)\right) d s\right)\right) d t\right)\right) \\
& \leq D\left(f(x), f\left(x_{1}\right)\right)+D\left(\sum _ { i = 1 } ^ { n } \int _ { a } ^ { x } k \left(\left(x, t, \tilde{F}_{1}\left(x, t, \tilde{u}_{i-1}(t)\right), G\left(t, \int_{a}^{t} \tilde{F}_{2}\left(t, s, \tilde{u}_{i-1}(s)\right) d s\right)\right) d t,\right.\right. \\
& \left.\sum_{i=1}^{n} \int_{a}^{x} k\left(\left(x_{1}, t, \tilde{F}_{1}\left(x_{1}, t, \tilde{u}_{i-1}(t)\right), G\left(t, \int_{a}^{t} \tilde{F}_{2}\left(t, s, \tilde{u}_{i-1}(s)\right) d s\right)\right) d t\right)\right) \\
& +D\left(\sum_{i=1}^{n} \int_{x}^{x_{1}} k\left(\left(x_{1}, t, \tilde{F}_{1}\left(x_{1}, t, \tilde{u}_{i-1}(t)\right), G\left(t, \int_{a}^{t} \tilde{F}_{2}\left(t, s, \tilde{u}_{i-1}(s)\right) d s\right)\right) d t\right), \tilde{0}\right) \\
& \leq D\left(f(x), f\left(x_{1}\right)\right)+\int_{a}^{x} D\left(\sum _ { i = 1 } ^ { n } k \left(x, t, \tilde{F}_{1}\left(x, t, \tilde{u}_{i-1}(t)\right), G\left(t, \int_{a}^{t} \tilde{F}_{2}\left(t, s, \tilde{u}_{i-1}(s)\right) d s\right),\right.\right. \\
& \sum_{i=1}^{n} \int_{a}^{x} k\left(\left(x_{1}, t, \tilde{F}_{1}\left(x_{1}, t, \tilde{u}_{i-1}(t)\right), G\left(t, \int_{a}^{t} \tilde{F}_{2}\left(t, s, \tilde{u}_{i-1}(s)\right) d s\right)\right)\right) d t \\
& +\int_{x}^{x_{1}} D\left(\sum_{i=1}^{n} k\left(\left(x_{1}, t, \tilde{F}_{1}\left(x_{1}, t, \tilde{u}_{i-1}(t)\right), G\left(t, \int_{a}^{t} \tilde{F}_{2}\left(t, s, \tilde{u}_{i-1}(s)\right) d s\right)\right), \tilde{0}\right) d t\right. \\
& \leq D\left(f(x), f\left(x_{1}\right)\right)+(b-a) \sup D\left[\sum _ { i = 1 } ^ { n } k \left(\left(x, t, \tilde{F}_{1}\left(x, t, \tilde{u}_{i-1}(t)\right), G\left(t, \int_{a}^{t} \tilde{F}_{2}\left(t, s, \tilde{u}_{i-1}(s)\right) d s\right)\right.\right.\right. \text {, } \\
& \sum_{i=1}^{n} k\left(\left(x_{1}, t, \tilde{F}_{1}\left(x_{1}, t, \tilde{u}_{i-1}(t)\right), G\left(t, \int_{a}^{t} \tilde{F}_{2}\left(t, s, \tilde{u}_{i-1}(s)\right) d s\right)\right]\right. \\
& +\left(x_{1}-x\right)\left[\sup D\left[\sum_{i=1}^{n} k\left(\left(x_{1}, t, \tilde{F}_{1}\left(x_{1}, t, \tilde{u}_{i-1}(t)\right), G\left(t, \int_{a}^{t} \tilde{F}_{2}\left(t, s, \tilde{u}_{i-1}(s)\right) d s\right)\right), \tilde{0}\right]\right]\right. \\
& D\left(\omega_{n}(x), \omega_{n}\left(x_{1}\right)\right) \rightarrow 0, x \rightarrow x_{1}
\end{aligned}
$$

and the sequence $\left\{\omega_{n}(x)\right\}_{n=0}^{\infty}$ is continuous on $[\mathrm{a}, \mathrm{b}]$

in this part we will prove the sequence $\left\{\omega_{n}(x)\right\}_{n=0}^{\infty}$ is convergent uniformly for $n \geq 0$ we get

$$
\begin{gathered}
D\left(\omega_{n+1}(x), \omega_{n}(x)\right)=D\left(f(x)+\sum_{i=1}^{n+1} \int_{a}^{x} k\left(\left(x, t, \tilde{F}_{1}\left(x, t, \tilde{u}_{i-1}(t)\right), G\left(t, \int_{a}^{t} \tilde{F}_{2}\left(t, s, \tilde{u}_{i-1}(s)\right) d s\right)\right) d t, \omega_{n}(x)\right)\right. \\
=D\left(f(x)+\sum_{i=1}^{n} \int_{a}^{x} k\left(\left(x, t, \tilde{F}_{1}\left(x, t, \tilde{u}_{i-1}(t)\right), G\left(t, \int_{a}^{t} \tilde{F}_{2}\left(t, s, \tilde{u}_{i-1}(s)\right) d s\right)\right) d t\right.\right. \\
\quad+\int_{a}^{x} k\left(\left(x, t, \tilde{F}_{1}\left(x, t, \tilde{u}_{n}(t)\right), G\left(t, \int_{a}^{t} \tilde{F}_{2}\left(t, s, \tilde{u}_{n}(s)\right) d s\right)\right) d t, \omega_{n}(x)\right) \\
=D\left(\omega_{n}(x)+\int_{a}^{x} k\left(\left(x, t, \tilde{F}_{1}\left(x, t, \tilde{u}_{n}(t)\right), G\left(t, \int_{a}^{t} \tilde{F}_{2}\left(t, s, \tilde{u}_{n}(s)\right) d s\right)\right) d t, \omega_{n}(x)\right)\right.
\end{gathered}
$$




$$
\begin{gathered}
=D\left(\int_{a}^{x} k\left(\left(x, t, \tilde{F}_{1}\left(x, t, \tilde{u}_{n}(t)\right), G\left(t, \int_{a}^{t} \tilde{F}_{2}\left(t, s, \tilde{u}_{n}(s)\right) d s\right)\right) d t, \tilde{0}\right)\right. \\
\leq \int_{a}^{x} D\left(k\left(\left(x, t, \tilde{F}_{1}\left(x, t, \tilde{u}_{n}(t)\right), G\left(t, \int_{a}^{t} \tilde{F}_{2}\left(t, s, \tilde{u}_{n}(s)\right) d s\right)\right), \tilde{0}\right) d t\right. \\
\leq(b-a)\left[\left(L+L_{1}\right) \sup D(x, 0)+(b-a)\left[\left(L+L_{1}+L_{2}\right)+(b-a) L_{3}\right) \sup D(t, \tilde{0})\right. \\
\left.\left.+(b-a) L_{3} \sup D(s, \tilde{0})+(b-a)\left[(b-a)\left(L_{3}+L\right) \sup D\left(\tilde{u}_{n}(t)\right), \tilde{0}\right)\right]\right]
\end{gathered}
$$

Now we obtain

$\sup D\left(\omega_{n+1}(x), \omega_{n}(x)\right) \leq(b-a)\left[\left(L+L_{1}\right) \sup D(x, 0)+(b-a)\left[\left(L+L_{1}+L_{2}\right)+(b-a) L_{3}\right) \sup D(t, \tilde{0})\right.$

$$
\left.\left.+(b-a) L_{3} \sup D(s, \tilde{0})+(b-a)\left[(b-a)\left(L_{3}+L\right) \sup D\left(\tilde{u}_{n}(t)\right), \tilde{0}\right)\right]\right]
$$$$
D\left(u_{n}(x), \tilde{0}\right)=D\left(\int_{a}^{x} k\left(\left(x, t, \tilde{F}_{1}\left(x, t, \tilde{u}_{n-1}(t)\right), G\left(t, \int_{a}^{t} \widetilde{F}_{2}\left(t, s, \tilde{u}_{n-1}(s)\right) d s\right)\right) d t, \tilde{0}\right)\right.
$$$$
\leq \int_{a}^{x} D\left(k\left(\left(x, t, \tilde{F}_{1}\left(x, t, \tilde{u}_{n-1}(t)\right), G\left(t, \int_{a}^{t} \tilde{F}_{2}\left(t, s, \tilde{u}_{n-1}(s)\right) d s\right)\right), \tilde{0}\right) d t\right.
$$

$\leq(b-a)\left[\left(L+L_{1}\right) \sup D(x, 0)+(b-a)\left[\left(L+L_{1}+L_{2}\right)+(b-a) L_{3}\right) \sup D(t, \tilde{0})\right.$

$$
\left.\left.+(b-a) L_{3} \sup D(s, \tilde{0})+(b-a)\left[(b-a)\left(L_{3}+L\right) \sup D\left(\tilde{u}_{n-1}(t)\right), \tilde{0}\right)\right]\right]
$$

$$
\begin{gathered}
D\left(u_{n-1}(x), \tilde{0}\right) \leq(b-a)\left[\left(L+L_{1}\right) \sup D(x, 0)+(b-a)\left[\left(L+L_{1}+L_{2}\right)+(b-a) L_{3}\right) \sup D(t, \tilde{0})\right. \\
\left.\left.+(b-a) L_{3} \sup D(s, \tilde{0})+(b-a)\left[(b-a)\left(L_{3}+L\right) \sup D\left(\tilde{u}_{n-2}(t)\right), \tilde{0}\right)\right]\right]
\end{gathered}
$$

we obtain

$$
\begin{gathered}
D\left(u_{n}(x), \tilde{0}\right) \leq(b-a)\left[\left(L+L_{1}\right) D(x, 0)+(b-a)\left[\left(L+L_{1}+L_{2}\right)+(b-a) L_{3}\right) D(t, \tilde{0})\right. \\
\left.\left.+(b-a) L_{3} D(s, \tilde{0})+(b-a)\left[(b-a)\left(L_{3}+L\right) D\left(\tilde{u}_{n-1}(t)\right), \tilde{0}\right)\right]\right] \\
\quad \leq\left[( b - a ) \left[\left(L+L_{1}\right) D(x, 0)+(b-a)\left[\left(L+L_{1}+L_{2}\right)+(b-a) L_{3}\right) D(t, \tilde{0})\right.\right. \\
\left.\left.\left.+(b-a) L_{3} D(s, \tilde{0})+(b-a)\left[(b-a)\left(L_{3}+L\right)\right]^{2} D\left(\tilde{u}_{n-2}(t)\right), \tilde{0}\right)\right]\right] \\
\leq\left[( b - a ) \left[\left(L+L_{1}\right) D(x, 0)+(b-a)\left[\left(L+L_{1}+L_{2}\right)+(b-a) L_{3}\right) D(t, \tilde{0})\right.\right. \\
\quad+(b-a) L_{3} D(s, \tilde{0})+(b-a)\left[(b-a)\left(L_{3}+L\right)\right]^{n} \sup D(f(x), \tilde{0}) \\
\quad D\left(\omega_{n+1}(x), \omega_{n}(x)\right) \leq \sup D\left(\omega_{n+1}(x), \omega_{n}(x)\right) \leq \\
{\left[( b - a ) \left[\left(L+L_{1}\right) D(x, 0)+(b-a)\left[\left(L+L_{1}+L_{2}\right)+(b-a) L_{3}\right) D(t, \tilde{0})\right.\right.} \\
+(b-a) L_{3} D(s, \tilde{0})+(b-a)\left[(b-a)\left(L_{3}+L\right)\right]^{n+1} \sup D(f(x), \tilde{0})
\end{gathered}
$$

the series $(b-a)\left[\left(L+L_{1}\right) D(x, 0)+(b-a)\left[\left(L+L_{1}+L_{2}\right)+(b-a) L_{3}\right) D(t, \tilde{0})\right.$

$+(b-a) L_{3} D(s, \tilde{0})+(b-a)\left[(b-a)\left(L_{3}+L\right)\right] \sup D(f(x), \tilde{0}) \sum_{n=0}^{\infty}\left[\left[(b-a)\left[\left(L+L_{1}\right) D(x, 0)\right.\right.\right.$

$+(b-a)\left[\left(L+L_{1}+L_{2}\right)+(b-a) L_{3}\right) D(t, \tilde{0})+(b-a) L_{3} D(s, \tilde{0})+(b-a)\left[(b-a)\left(L_{3}+L\right)\right]^{n}$

the series $\sum_{n=0}^{\infty} D\left(\omega_{n+1}(x), \omega_{n}(x)\right)$ is uniformly convergent on [a,b], and the uniformly convergent of the sequence $\left\{\omega_{n}(x)\right\}_{n=0}^{\infty}$. if denoted $u(x)=\lim _{n \rightarrow \infty} \omega_{n}(x)$, then $\mathrm{u}(\mathrm{x})$ is satisfy all the conditions for theorem (2.1) 
We prove the uniqueness of solution $>$ Let $\mathrm{u}(\mathrm{x})$ and $u^{*}(x)$ are two continuous solution of $\mathrm{Eq}(7)$ on $[\mathrm{a}, \mathrm{b}]$

$$
D\left(\tilde{u}(x), \tilde{u}^{*}(x)\right) \geq 0
$$

and

$$
\begin{gathered}
D\left(\tilde{u}(x), \tilde{u}^{*}(x)\right)=D\left(\tilde{u}(x)+\omega_{n}(x), \tilde{u}^{*}(x)+\omega_{n}(x)\right) \\
\leq D\left(\tilde{u}(x), \omega_{n}(x)\right)+D\left(\tilde{u}^{*}(x), \omega_{n}(x)\right) \\
D\left(\tilde{u}(x), \omega_{n}(x)\right) \rightarrow 0 \\
D\left(\tilde{u}^{*}(x), \omega_{n}(x)\right) \rightarrow 0
\end{gathered}
$$

when $n \rightarrow \infty$, then $D\left(\tilde{u}(x), \tilde{u}^{*}(x)\right)=0$, then $\tilde{u}(x)=\tilde{u}^{*}(x)$

\section{2. numerical example}

In this prrt we will discuss case of new formula by using homotopy analysis method.

And comparing the approximate method with he known exact solution and calculate the absolute error between the exact and approximate. Also give some finger for all cases.

$k\left(x, t, F_{1 \alpha}(t, u(t, \alpha))\right) \geq 0, \overline{k\left(x, t, G\left(t, \int_{a}^{t} F_{2 \alpha}(t, s, u(s, \alpha)) d s\right)\right.} \geq 0, k\left(x, t, \overline{G\left(t, \int_{a}^{t} F_{2 \alpha}(t, s, u(s, \alpha)) d s\right)}<0\right.$

$\underline{k\left(x, t, F_{1 \alpha}(t, u(t, \alpha))\right)}<0,0 \leq x \leq t \leq b, a \leq s \leq t$

$$
\begin{aligned}
& \underline{u}(x, \alpha)=\underline{f}(x, \alpha)+\lambda \int_{a}^{c} k\left(x, t, \underline{F}_{1 \alpha}(x, t, \underline{u}(t, \alpha)) d t+\lambda \int_{c}^{x} k\left(x, t, G\left(t, \int_{a}^{t} \bar{F}_{2 \alpha}(t, s, \bar{u}(s, \alpha)) d s\right) d t\right.\right. \\
& \bar{u}(x, \alpha)=\bar{f}(x, \alpha)+\lambda \int_{a}^{c} k\left(x, t, G\left(t, \int_{a}^{t} \bar{F}_{2 \alpha}(t, s, \bar{u}(s, \alpha)) d s\right) d t+\lambda \int_{c}^{x} k\left(x, t, \underline{F}_{1 \alpha}(x, t, \underline{u}(t, \alpha)) d t\right.\right.
\end{aligned}
$$

\section{Example 1:}

Now we discus this example

We will discuss Now the case of formula $2, k\left(x, t, F_{1 \alpha}(t, u(t, \alpha))\right) \geq 0, G \overline{\left(t, \int_{a}^{t} F_{2 \alpha}(t, s, u(s, \alpha)) d s\right)} \geq 0$

And $\underline{k\left(x, t, F_{1 \alpha}(t, u(t, \alpha))\right)}<0, G \overline{\left(t, \int_{a}^{t} F_{2 \alpha}(t, s, U(s, \alpha)) d s\right)}<0$

$\underline{u}(x, \alpha)=\underline{f}(x, \alpha)+\lambda \int_{a}^{c} k\left(x, t, \underline{F}_{1 \alpha}(t, \underline{u}(t, \alpha))\right) d t+\lambda \int_{c}^{x} k\left(x, t, G\left(t, \int_{a}^{t} \bar{F}_{2 \alpha}(t, s, \bar{u}(s, \alpha)) d s\right) d t\right.$

$\bar{u}(x, \alpha)=\bar{f}(x, \alpha)+\lambda \int_{a}^{c} k\left(x, t, G\left(t, \int_{a}^{t} \bar{F}_{2 \alpha}(t, s, \bar{u}(s, \alpha)) d s\right) d t+\lambda \int_{c}^{x} k\left(x, t, \underline{F}_{1 \alpha}(t, \underline{u}(t, \alpha))\right) d t\right.$

Where

$$
\begin{gathered}
k\left(x, t, \underline{F}_{1 \alpha}(t, \underline{u}(t, \alpha))\right)=x t(\underline{u}(x, \alpha))^{2} \\
k\left(x, t, G\left(t, \int_{a}^{t} \bar{F}_{2 \alpha}(t, s, \bar{u}(s, \alpha)) d s\right)=x t \int_{a}^{t}(\bar{u}(s, \alpha))^{2} d s\right.
\end{gathered}
$$

$\underline{f}(x, \alpha)=\mathrm{x}^{\wedge} 2 * \mathrm{r}-(1 / 5){ }^{*} \mathrm{x}^{\wedge} 6{ }^{*} \mathrm{r}^{\wedge} 2-(1 / 30)^{*} \mathrm{x}^{\wedge} 6 *(2-\mathrm{r})^{\wedge} 2$

$\bar{f}(x, \alpha)=\mathrm{x}^{\wedge} 2 *(2-\mathrm{r})-(1 / 5)^{*} \mathrm{x}^{\wedge} 6^{*} \mathrm{r}^{\wedge} 2-(1 / 30)^{*} \mathrm{x}^{\wedge} 6^{*}(2-\mathrm{r})^{\wedge} 2$ 
the intimal condition is $\underline{u}_{0}(x, \alpha)=\bar{u}_{0}(x, \alpha)=0$, and $0 \leq \alpha \leq 1$

the exact solution $\underline{u}(x, \alpha)=x^{2} \alpha$ and $\bar{u}(x, \alpha)=x^{2}(2-\alpha)$

$$
\underline{u}_{0}(x, \alpha)=0
$$

$\underline{u}_{1}(x, \alpha)=-\mathrm{h}^{*}\left(\mathrm{x}^{\wedge} 2^{*} \mathrm{r}-(1 / 5)^{*} \mathrm{x}^{\wedge} 6^{*} \mathrm{r}^{\wedge} 2-(1 / 30)^{*} \mathrm{x}^{\wedge} 6^{*}(2-\mathrm{r})^{\wedge} 2\right)$

$$
\underline{u}_{2}(x, \alpha)=(1+h) \underline{u}_{1}(x, \alpha)-h \lambda\left[\int_{0}^{c} \overline{\boldsymbol{R}}_{1}(x, t ; \alpha) d t+\int_{c}^{x} \underline{\boldsymbol{R}}_{1}(x, t ; \alpha) d t\right]
$$

$=-(1+\mathrm{h}) * \mathrm{~h}^{*}\left(\mathrm{x}^{\wedge} 2^{*} \mathrm{r}-(1 / 5)^{*} \mathrm{x}^{\wedge} 6^{*} \mathrm{r}^{\wedge} 2-(1 / 30)^{*} \mathrm{x}^{\wedge} 6 *(2-\mathrm{r})^{\wedge} 2\right)-\mathrm{h}^{*}\left(\mathrm{x}^{*} \mathrm{~h}^{\wedge} 2^{*}\left(0.9390024038 \mathrm{e}-5^{*}\left(-(1 / 5) * \mathrm{r}^{\wedge} 2-(1 / 30)^{*}(2-\right.\right.\right.$ $\left.\left.\mathrm{r})^{\wedge} 2\right)^{\wedge} 2+0.4340277778 \mathrm{e}-3^{*} \mathrm{r}^{*}\left(-(1 / 5)^{*} \mathrm{r}^{\wedge} 2-(1 / 30)^{*}(2-\mathrm{r})^{\wedge} 2\right)+0.6250000000 \mathrm{e}-2^{*} \mathrm{r}^{\wedge} 2\right)+\mathrm{h}^{\wedge} 2^{*}\left((1 / 182)^{*}\left(-(1 / 5)^{*} \mathrm{r}^{\wedge} 2-\right.\right.$ $\left.\left.(1 / 30)^{*}(2-r)^{\wedge} 2\right)^{\wedge} 2 * x^{\wedge} 14+\left(1 / 45^{*}(2-r)\right)^{*}\left(-(1 / 5)^{*} r^{\wedge} 2-(1 / 30)^{*}(2-r) \wedge 2\right)^{*} x^{\wedge} 10+(1 / 30)^{*} x^{\wedge} 6 *(2-r)^{\wedge} 2\right)-$ $\mathrm{h}^{\wedge} 2^{*}\left(3.353580014^{*} 10^{\wedge}(-7)^{*}\left(-(1 / 5)^{*} \mathrm{r}^{\wedge} 2-(1 / 30)^{*}(2-\mathrm{r})^{\wedge} 2\right)^{\wedge} 2+(0.2170138889 \mathrm{e}-4 *(2-\mathrm{r}))^{*}\left(-(1 / 5)^{*} \mathrm{r}^{\wedge} 2-(1 / 30)^{*}(2-\right.\right.$ r) $\left.\left.\left.)^{\wedge} 2\right)+0.5208333333 e-3^{*}(2-r)^{\wedge} 2\right)\right)$

$\underline{u}_{3}(x, \alpha)=(1+\mathrm{h}) *\left(-(1+\mathrm{h}) * \mathrm{~h}^{*}\left(\mathrm{x}^{\wedge} 2^{*} \mathrm{r}-(1 / 5) * \mathrm{x}^{\wedge} 6^{*} \mathrm{r}^{\wedge} 2-(1 / 30) * \mathrm{x}^{\wedge} 6^{*}(2-\mathrm{r})^{\wedge} 2\right)-\right.$ $\mathrm{h}^{*}\left(\mathrm{x}^{*} \mathrm{~h}^{\wedge} 2^{*}\left(0.9390024038 \mathrm{e}-5^{*}\left(-(1 / 5)^{*} \mathrm{r}^{\wedge} 2-(1 / 30) *(2-\mathrm{r})^{\wedge} 2\right)^{\wedge} 2+0.4340277778 \mathrm{e}-3^{*} \mathrm{r}^{*}(-\right.\right.$ $\left.\left.(1 / 5) * r^{\wedge} 2-(1 / 30) *(2-r)^{\wedge} 2\right)+0.6250000000 e-2^{*} r^{\wedge} 2\right)+h^{\wedge} 2^{*}\left((1 / 182) *\left(-(1 / 5)^{*} r^{\wedge} 2-(1 / 30) *(2-\right.\right.$ $\left.\left.r)^{\wedge} 2\right)^{\wedge} 2^{*} x^{\wedge} 14+\left(1 / 45^{*}(2-r)\right)^{*}\left(-(1 / 5)^{*} r^{\wedge} 2-(1 / 30) *(2-r)^{\wedge} 2\right)^{*} x^{\wedge} 10+(1 / 30)^{*} x^{\wedge} 6^{*}(2-r)^{\wedge} 2\right)-$ $\mathrm{h}^{\wedge} 2^{*}\left(3.353580014^{*} 10^{\wedge}(-7) *\left(-(1 / 5)^{*} \mathrm{r}^{\wedge} 2-(1 / 30) *(2-\mathrm{r})^{\wedge} 2\right)^{\wedge} 2+(0.2170138889 \mathrm{e}-4 *(2-\mathrm{r}))^{*}(-\right.$ $\left.\left.\left.\left.(1 / 5)^{*} \mathrm{r}^{\wedge} 2-(1 / 30)^{*}(2-\mathrm{r})^{\wedge} 2\right)+0.5208333333 \mathrm{e}-3^{*}(2-\mathrm{r})^{\wedge} 2\right)\right)\right)-\mathrm{h}^{*}\left(2.548432250^{*} 10^{\wedge}(-\right.$ $11)^{*} h^{\wedge} 6^{*} \mathrm{r}^{\wedge} 2^{*}\left(-.2000000000^{*} \mathrm{r}^{\wedge} 2-0.3333333333 \mathrm{e}-1^{*}\left(2 .-1{ }^{*} \mathrm{r}\right)^{\wedge} 2\right)^{\wedge} 2+0.5259697569 \mathrm{e}-$ $4^{*} \mathrm{~h}^{\wedge} 6^{*} \mathrm{r}^{*}\left(-.2000000000^{*} \mathrm{r}^{\wedge} 2-0.3333333333 \mathrm{e}-1^{*}\left(2 .-1{ }^{*} \mathrm{r}\right)^{\wedge} 2\right)^{\wedge} 3^{*} \mathrm{x}^{\wedge} 26+9.781275041^{*} 10^{\wedge}(-$ $9)^{*} \mathrm{~h}^{\wedge} 4^{*}\left(\mathrm{~h}^{\wedge} 2^{*}\left(0.9390024038 \mathrm{e}-5^{*}\left(-.2000000000^{*} \mathrm{r}^{\wedge} 2-0.3333333333 \mathrm{e}-1 *(2 .-\right.\right.\right.$

$\left.\left.1{ }^{*} \mathrm{r}\right)^{\wedge} 2\right)^{\wedge} 2+\left(0.4340277778 \mathrm{e}-3^{*}(2 .-1 . * \mathrm{r})\right)^{*}\left(-.2000000000^{*} \mathrm{r}^{\wedge} 2-0.3333333333 \mathrm{e}-1^{*}(2 .-\right.$ $\left.\left.\left.1 .^{*} \mathrm{r}\right)^{\wedge} 2\right)+0.6250000000 \mathrm{e}-2^{*}\left(2 .-1 .^{*} \mathrm{r}\right)^{\wedge} 2\right)-1{ }^{*} \mathrm{x}^{*} \mathrm{~h}^{\wedge} 2^{*}\left(0.9390024038 \mathrm{e}-5^{*}\left(-.2000000000^{*} \mathrm{r}^{\wedge} 2-\right.\right.$ $\left.0.3333333333 \mathrm{e}-1^{*}\left(2 .-1{ }^{*} \mathrm{r}\right)^{\wedge} 2\right)^{\wedge} 2+0.4340277778 \mathrm{e}-3^{*} \mathrm{r}^{*}\left(-.2000000000^{*} \mathrm{r}^{\wedge} 2-0.3333333333 \mathrm{e}-\right.$ $\left.\left.\left.1^{*}\left(2 .-1{ }^{*} \mathrm{r}\right)^{\wedge} 2\right)+0.6250000000 \mathrm{e}-2^{*} \mathrm{r}^{\wedge} 2\right)\right)^{*}\left(-.2000000000^{*} \mathrm{r}^{\wedge} 2-0.3333333333 \mathrm{e}-1^{*}\left(2 .-1 *^{*} \mathrm{r}\right)^{\wedge} 2\right)^{\wedge} 2-$ $\left(0.2170138889 \mathrm{e}-4^{*}(1 .+\mathrm{h})\right)^{*} \mathrm{~h}^{*}\left(2 .-1 *^{*} \mathrm{r}\right) *\left(-\left(1 *^{*}(1 .+\mathrm{h})\right)^{*} \mathrm{~h}^{*}\left(-.2000000000^{*} \mathrm{r}^{\wedge} 2-0.3333333333 \mathrm{e}-\right.\right.$ $\left.\left.1^{*}\left(2 .-1{ }^{*} \mathrm{r}\right)^{\wedge} 2\right)-.2000000000^{*} \mathrm{~h}^{\wedge} 3^{*} \mathrm{r}^{\wedge} 2\right)+0.3333333333 \mathrm{e}-1^{*}(1 .+\mathrm{h})^{\wedge} 2^{*} \mathrm{~h}^{\wedge} 2^{*}(2 .-$

$1 . * r)^{\wedge} 2^{*} \mathrm{x}^{\wedge} 6+1.917897066^{*} 10^{\wedge}(-9)^{*}(1 .+\mathrm{h}) * \mathrm{~h}^{\wedge} 4 *\left(2 .-1{ }^{*} \mathrm{r}\right)^{*}\left(-.2000000000^{*} \mathrm{r}^{\wedge} 2-0.3333333333 \mathrm{e}-\right.$ $\left.1^{*}\left(2 .-1 *^{*} \mathrm{r}\right)^{\wedge} 2\right)^{\wedge} 2-5.540591526^{*} 10^{\wedge}(-9)^{*}\left(-\left(1 \text { * }^{*}(1 .+\mathrm{h})\right)^{*} \mathrm{~h}^{*}\left(-.2000000000^{*} \mathrm{r}^{\wedge} 2-0.3333333333 \mathrm{e}-\right.\right.$ $\left.\left.1^{*}\left(2 .-1{ }^{*} \mathrm{r}\right)^{\wedge} 2\right)-.2000000000^{*} \mathrm{~h}^{\wedge} 3^{*} \mathrm{r}^{\wedge} 2\right)^{*} \mathrm{~h}^{\wedge} 3^{*} \mathrm{r}^{*}\left(-.2000000000^{*} \mathrm{r}^{\wedge} 2-0.3333333333 \mathrm{e}-1^{*}(2 .-\right.$ $\left.\left.1{ }^{*} \mathrm{r}\right)^{\wedge} 2\right)-7.939346623^{*} 10^{\wedge}(-11)^{*}\left(-\left(1 .^{*}(1 .+\mathrm{h})\right)^{*} \mathrm{~h}^{*}\left(-.2000000000^{*} \mathrm{r}^{\wedge} 2-0.3333333333 \mathrm{e}-1^{*}(2 .-\right.\right.$ $\left.\left.\left.1{ }^{*} \mathrm{r}\right)^{\wedge} 2\right)-.2000000000^{*} \mathrm{~h}^{\wedge} 3^{*} \mathrm{r}^{\wedge} 2\right)^{*} \mathrm{~h}^{\wedge} 3^{*}\left(-.2000000000^{*} \mathrm{r}^{\wedge} 2-0.3333333333 \mathrm{e}-1^{*}(2 .-\right.$ $\left.\left.1{ }^{*} \mathrm{r}\right)^{\wedge} 2\right)^{\wedge} 2+8.220223064 * 10^{\wedge}(-7)^{*} \mathrm{~h}^{\wedge} 4^{*}\left(\mathrm{~h}^{\wedge} 2^{*}\left(0.9390024038 \mathrm{e}-5^{*}\left(-.2000000000^{*} \mathrm{r}^{\wedge} 2-\right.\right.\right.$ $\left.0.3333333333 \mathrm{e}-1^{*}\left(2 .-1 *^{*} \mathrm{r}\right)^{\wedge} 2\right)^{\wedge} 2+\left(0.4340277778 \mathrm{e}-3^{*}\left(2 .-1{ }^{*} \mathrm{r}\right)\right)^{*}\left(-.2000000000^{*} \mathrm{r}^{\wedge} 2-\right.$ $\left.\left.0.3333333333 \mathrm{e}-1^{*}\left(2 .-1{ }^{*} \mathrm{r}\right)^{\wedge} 2\right)+0.6250000000 \mathrm{e}-2^{*}\left(2 .-1{ }^{*} \mathrm{r}\right)^{\wedge} 2\right)-1{ }^{*} \mathrm{x}^{*} \mathrm{~h}^{\wedge} 2^{*}\left(0.9390024038 \mathrm{e}-5^{*}(-\right.$ $\left..2000000000^{*} r^{\wedge} 2-0.3333333333 \mathrm{e}-1^{*}\left(2 .-1 *^{*} \mathrm{r}\right)^{\wedge} 2\right)^{\wedge} 2+0.4340277778 \mathrm{e}-3^{*} \mathrm{r}^{*}\left(-.2000000000^{*} \mathrm{r}^{\wedge} 2-\right.$ $\left.\left.\left.0.3333333333 \mathrm{e}-1^{*}\left(2 .-1 .^{*} \mathrm{r}\right)^{\wedge} 2\right)+0.6250000000 \mathrm{e}-2^{*} \mathrm{r}^{\wedge} 2\right)\right)^{*} \mathrm{r}^{*}\left(-.2000000000^{*} \mathrm{r}^{\wedge} 2-\right.$ $\left.0.3333333333 \mathrm{e}-1 *\left(2 .-1 *^{*} \mathrm{r}\right)^{\wedge} 2\right)+\left(0.5494505494 \mathrm{e}-2^{*}\left((.4444444444 *(1 .+\mathrm{h}))^{*} \mathrm{~h}^{\wedge} 4 *\left(2 .-1{ }^{*} \mathrm{r}\right)^{*} \mathrm{r}^{*}(-\right.\right.$ $\left..2000000000^{*} \mathrm{r}^{\wedge} 2-0.3333333333 \mathrm{e}-1^{*}\left(2 .-1 .^{*} \mathrm{r}\right)^{\wedge} 2\right)+\left(-\left(1 *^{*}(1 .+\mathrm{h})\right)^{*} \mathrm{~h}^{*}\left(-.2000000000^{*} \mathrm{r}^{\wedge} 2-\right.\right.$ $\left.\left.\left.\left.0.3333333333 \mathrm{e}-1^{*}(2 .-1 . * \mathrm{r})^{\wedge} 2\right)-.2000000000 * \mathrm{~h}^{\wedge} 3^{*} \mathrm{r}^{\wedge} 2\right)^{\wedge} 2\right)\right)^{*} \mathrm{x}^{\wedge} 14+0.1041666667 \mathrm{e}-$

$\underline{U}(x, \alpha)=\quad-h^{*}\left(x^{\wedge} 2^{*} r-(1 / 5)^{*} x^{\wedge} 6^{*} r^{\wedge} 2-(1 / 30)^{*} x^{\wedge} 6^{*}(2-r)^{\wedge} 2\right)-(1+h)^{*} h^{*}\left(x^{\wedge} 2^{*} r-(1 / 5)^{*} x^{\wedge} 6^{*} r^{\wedge} 2-(1 / 30)^{*} x^{\wedge} 6^{*}(2-r)^{\wedge} 2\right)-$ $\mathrm{h}^{*}\left(\mathrm{x}^{*} \mathrm{~h}^{\wedge} 2^{*}\left(0.9390024038 \mathrm{e}-5^{*}\left(-(1 / 5)^{*} \mathrm{r}^{\wedge} 2-(1 / 30)^{*}(2-\mathrm{r})^{\wedge} 2\right)^{\wedge} 2+0.4340277778 \mathrm{e}-3^{*} \mathrm{r}^{*}\left(-(1 / 5)^{*} \mathrm{r}^{\wedge} 2-(1 / 30)^{*}(2-\right.\right.\right.$ $\left.\left.\mathrm{r})^{\wedge} 2\right)+0.6250000000 \mathrm{e}-2^{*} \mathrm{r}^{\wedge} 2\right)+\mathrm{h}^{\wedge} 2^{*}\left((1 / 182)^{*}\left(-(1 / 5)^{*} \mathrm{r}^{\wedge} 2-(1 / 30)^{*}(2-\mathrm{r})^{\wedge} 2\right)^{\wedge} 2^{*} \mathrm{x}^{\wedge} 14+(1 / 45 *(2-\mathrm{r}))^{*}\left(-(1 / 5) * \mathrm{r}^{\wedge} 2-\right.\right.$ $\left.\left.(1 / 30)^{*}(2-r)^{\wedge} 2\right)^{*} x^{\wedge} 10+(1 / 30)^{*} x^{\wedge} 6^{*}(2-r)^{\wedge} 2\right)-h^{\wedge} 2^{*}\left(3.353580014 * 10^{\wedge}(-7)^{*}\left(-(1 / 5)^{*} r^{\wedge} 2-(1 / 30)^{*}(2-\right.\right.$ $\left.\left.\left.\mathrm{r})^{\wedge} 2\right)^{\wedge} 2+\left(0.2170138889 \mathrm{e}-4^{*}(2-\mathrm{r})\right)^{*}\left(-(1 / 5) * \mathrm{r}^{\wedge} 2-(1 / 30)^{*}(2-\mathrm{r})^{\wedge} 2\right)+0.5208333333 \mathrm{e}-3 *(2-\mathrm{r})^{\wedge} 2\right)\right)+=$ $(1+h) * h^{*}\left(x^{\wedge} 2^{*} r-(1 / 5) * x^{\wedge} 6^{*} r^{\wedge} 2-(1 / 30) * x^{\wedge} 6 *(2-r) \wedge 2\right)-h^{*}\left(x^{*} h^{\wedge} 2^{*}(0.9390024038 e-5 *(-\right.$ $\left.(1 / 5) * r^{\wedge} 2-(1 / 30) *(2-r)^{\wedge} 2\right)^{\wedge} 2+0.4340277778 \mathrm{e}-3^{*} r^{*}\left(-(1 / 5) * r^{\wedge} 2-(1 / 30) *(2-\right.$ $\left.\left.r)^{\wedge} 2\right)+0.6250000000 e-2^{*} r^{\wedge} 2\right)+h^{\wedge} 2^{*}\left((1 / 182)^{*}\left(-(1 / 5)^{*} r^{\wedge} 2-(1 / 30)^{*}(2-r)^{\wedge} 2\right)^{\wedge} 2^{*} x^{\wedge} 14+\left(1 / 45^{*}(2-\right.\right.$ $\left.r))^{*}\left(-(1 / 5)^{*} r^{\wedge} 2-(1 / 30) *(2-r)^{\wedge} 2\right)^{*} x^{\wedge} 10+(1 / 30)^{*} x^{\wedge} 6^{*}(2-r)^{\wedge} 2\right)-h^{\wedge} 2^{*}\left(3.353580014^{*} 10^{\wedge}(-7)^{*}(-\right.$ 
$\left.(1 / 5) * r^{\wedge} 2-(1 / 30) *(2-r)^{\wedge} 2\right)^{\wedge} 2+(0.2170138889 e-4 *(2-r))^{*}\left(-(1 / 5)^{*} r^{\wedge} 2-(1 / 30) *(2-\right.$ $\left.\left.\left.\left.r)^{\wedge} 2\right)+0.5208333333 \mathrm{e}-3^{*}(2-r)^{\wedge} 2\right)\right)\right)-h^{*}\left(2.548432250^{*} 10^{\wedge}(-11)^{*} h^{\wedge} 6^{*} \mathrm{r}^{\wedge} 2^{*}\left(-.2000000000^{*} \mathrm{r}^{\wedge} 2-\right.\right.$ $\left.0.3333333333 \mathrm{e}-1^{*}\left(2 .-1{ }^{*} \mathrm{r}\right)^{\wedge} 2\right)^{\wedge} 2+0.5259697569 \mathrm{e}-4^{*} \mathrm{~h}^{\wedge} 6^{*} \mathrm{r}^{*}\left(-.2000000000^{*} \mathrm{r}^{\wedge} 2-\right.$ $\left.0.3333333333 \mathrm{e}-1^{*}\left(2 .-1 *^{*} \mathrm{r}\right)^{\wedge} 2\right)^{\wedge} 3^{*} \mathrm{x}^{\wedge} 26+9.781275041^{*} 10^{\wedge}(-9)^{*} \mathrm{~h}^{\wedge} 4^{*}\left(\mathrm{~h}^{\wedge} 2^{*}(0.9390024038 \mathrm{e}-\right.$ $5^{*}\left(-.2000000000^{*} \mathrm{r}^{\wedge} 2-0.3333333333 \mathrm{e}-1^{*}\left(2 .-1{ }^{*} \mathrm{r}\right)^{\wedge} 2\right)^{\wedge} 2+\left(0.4340277778 \mathrm{e}-3^{*}\left(2 .-1{ }^{*} \mathrm{r}\right)\right)^{*}(-$ $\left.\left..2000000000^{*} r^{\wedge} 2-0.3333333333 \mathrm{e}-1^{*}\left(2 .-1 *^{*} \mathrm{r}\right)^{\wedge} 2\right)+0.6250000000 \mathrm{e}-2^{*}\left(2 .-1{ }^{*} \mathrm{r}\right)^{\wedge} 2\right)-$ $1{ }^{*} \mathrm{x}^{*} \mathrm{~h}^{\wedge} \mathrm{2}^{*}\left(0.9390024038 \mathrm{e}-5^{*}\left(-.2000000000^{*} \mathrm{r}^{\wedge} 2-0.3333333333 \mathrm{e}-1 *(2 .-\right.\right.$

$\left.\left.1{ }^{*} \mathrm{r}\right)^{\wedge} 2\right)^{\wedge} 2+0.4340277778 \mathrm{e}-3^{*} \mathrm{r}^{*}\left(-.2000000000^{*} \mathrm{r}^{\wedge} 2-0.3333333333 \mathrm{e}-1^{*}(2 .-\right.$

$\left.\left.\left.\left.1{ }^{*} \mathrm{r}\right)^{\wedge} 2\right)+0.6250000000 \mathrm{e}-2^{*} \mathrm{r}^{\wedge} 2\right)\right)^{*}\left(-.2000000000^{*} \mathrm{r}^{\wedge} 2-0.3333333333 \mathrm{e}-1^{*}(2 .-1 . * \mathrm{r})^{\wedge} 2\right)^{\wedge} 2-$ $\left(0.2170138889 \mathrm{e}-4^{*}(1 .+\mathrm{h})\right)^{*} \mathrm{~h}^{*}\left(2 .-1 *^{*} \mathrm{r}\right) *\left(-\left(1 *^{*}(1 .+\mathrm{h})\right)^{*} \mathrm{~h}^{*}\left(-.2000000000^{*} \mathrm{r}^{\wedge} 2-0.3333333333 \mathrm{e}-\right.\right.$ $\left.\left.1^{*}\left(2 .-1{ }^{*} \mathrm{r}\right)^{\wedge} 2\right)-.2000000000^{*} \mathrm{~h}^{\wedge} 3^{*} \mathrm{r}^{\wedge} 2\right)+0.3333333333 \mathrm{e}-1^{*}(1 .+\mathrm{h})^{\wedge} 2^{*} \mathrm{~h}^{\wedge} 2^{*}(2 .-$

$\left.1{ }^{*} \mathrm{r}\right)^{\wedge} 2^{*} \mathrm{x}^{\wedge} 6+1.917897066^{*} 10^{\wedge}(-9)^{*}(1 .+\mathrm{h})^{*} \mathrm{~h}^{\wedge} 4^{*}\left(2 .-1{ }^{*} \mathrm{r}\right) *\left(-.2000000000^{*} \mathrm{r}^{\wedge} 2-0.3333333333 \mathrm{e}-\right.$ $\left.1^{*}\left(2 .-1 *^{*} \mathrm{r}\right)^{\wedge} 2\right)^{\wedge} 2-5.540591526^{*} 10^{\wedge}(-9)^{*}\left(-\left(1 *^{*}(1 .+\mathrm{h})\right)^{*} \mathrm{~h}^{*}\left(-.2000000000^{*} \mathrm{r}^{\wedge} 2-0.3333333333 \mathrm{e}-\right.\right.$ $\left.\left.1^{*}\left(2 .-1{ }^{*} \mathrm{r}\right)^{\wedge} 2\right)-.2000000000^{*} \mathrm{~h}^{\wedge} 3^{*} \mathrm{r}^{\wedge} 2\right)^{*} \mathrm{~h}^{\wedge} 3^{*} \mathrm{r}^{*}\left(-.2000000000^{*} \mathrm{r}^{\wedge} 2-0.3333333333 \mathrm{e}-1^{*}(2 .-\right.$ $\left.\left.1{ }^{*} \mathrm{r}\right)^{\wedge} 2\right)-7.939346623^{*} 10^{\wedge}(-11)^{*}\left(-\left(1{ }^{*}(1 .+\mathrm{h})\right)^{*} \mathrm{~h}^{*}\left(-.2000000000^{*} \mathrm{r}^{\wedge} 2-0.3333333333 \mathrm{e}-1^{*}(2 .-\right.\right.$ 1. $\left.\left.\left.{ }^{*} \mathrm{r}\right)^{\wedge} 2\right)-.2000000000^{*} \mathrm{~h}^{\wedge} 3^{*} \mathrm{r}^{\wedge} 2\right)^{*} \mathrm{~h}^{\wedge} 3^{*}\left(-.2000000000^{*} \mathrm{r}^{\wedge} 2-0.3333333333 \mathrm{e}-1^{*}(2 .-\right.$ $\left.\left.1 *^{*} \mathrm{r}\right)^{\wedge} 2\right)^{\wedge} 2+8.220223064^{*} 10^{\wedge}(-7)^{*} \mathrm{~h}^{\wedge} 4^{*}\left(\mathrm{~h}^{\wedge} 2^{*}\left(0.9390024038 \mathrm{e}-5^{*}\left(-.2000000000^{*} \mathrm{r}^{\wedge} 2-\right.\right.\right.$ $\left.0.3333333333 \mathrm{e}-1^{*}\left(2 .-1 *^{*} \mathrm{r}\right)^{\wedge} 2\right)^{\wedge} 2+\left(0.4340277778 \mathrm{e}-3 *\left(2 .-1{ }^{*} \mathrm{r}\right)\right)^{*}\left(-.2000000000^{*} \mathrm{r}^{\wedge} 2-\right.$ $\left.\left.0.3333333333 \mathrm{e}-1^{*}\left(2 .-1 .^{*} \mathrm{r}\right)^{\wedge} 2\right)+0.6250000000 \mathrm{e}-2^{*}\left(2 .-1{ }^{*} \mathrm{r}\right)^{\wedge} 2\right)-1{ }^{*} \mathrm{x}^{*} \mathrm{~h}^{\wedge} 2^{*}\left(0.9390024038 \mathrm{e}-5^{*}(-\right.$ $\left..2000000000^{*} \mathrm{r}^{\wedge} 2-0.3333333333 \mathrm{e}-1^{*}\left(2 .-1{ }^{*} \mathrm{r}\right)^{\wedge} 2\right)^{\wedge} 2+0.4340277778 \mathrm{e}-3^{*} \mathrm{r}^{*}\left(-.2000000000^{*} \mathrm{r}^{\wedge} 2-\right.$ $\left.\left.\left.0.3333333333 \mathrm{e}-1^{*}\left(2 .-1 *^{*} \mathrm{r}\right)^{\wedge} 2\right)+0.6250000000 \mathrm{e}-2^{*} \mathrm{r}^{\wedge} 2\right)\right)^{*} \mathrm{r}^{*}\left(-.2000000000^{*} \mathrm{r}^{\wedge} 2-\right.$ $\left.0.3333333333 \mathrm{e}-1 *\left(2 .-1 *^{*} \mathrm{r}\right)^{\wedge} 2\right)+\left(0.5494505494 \mathrm{e}-2^{*}\left((.4444444444 *(1 .+\mathrm{h}))^{*} \mathrm{~h}^{\wedge} 4 *\left(2 .-1{ }^{*} \mathrm{r}\right)^{*} \mathrm{r}^{*}(-\right.\right.$ $\left..2000000000^{*} r^{\wedge} 2-0.3333333333 \mathrm{e}-1^{*}\left(2 .-1 .^{*} \mathrm{r}\right)^{\wedge} 2\right)+\left(-\left(1 *^{*}(1 .+\mathrm{h})\right)^{*} \mathrm{~h}^{*}\left(-.2000000000^{*} \mathrm{r}^{\wedge} 2-\right.\right.$ $\left.\left.\left.\left.0.3333333333 \mathrm{e}-1^{*}\left(2 .-1{ }^{*} \mathrm{r}\right)^{\wedge} 2\right)-.2000000000^{*} \mathrm{~h}^{\wedge} 3^{*} \mathrm{r}^{\wedge} 2\right)^{\wedge} 2\right)\right)^{*} \mathrm{x}^{\wedge} 14+0.1041666667 \mathrm{e}-$ $1^{*} \mathrm{~h}^{\wedge} 2^{*}\left(\mathrm{~h}^{\wedge} 2^{*}\left(0.9390024038 \mathrm{e}-5^{*}\left(-.2000000000^{*} \mathrm{r}^{\wedge} 2-0.3333333333 \mathrm{e}-1^{*}(2 .-\right.\right.\right.$

$\left.\left.1{ }^{*} \mathrm{r}\right)^{\wedge} 2\right)^{\wedge} 2+\left(0.4340277778 \mathrm{e}-3^{*}\left(2 .-1{ }^{*} \mathrm{r}\right)\right)^{*}\left(-.2000000000^{*} \mathrm{r}^{\wedge} 2-0.3333333333 \mathrm{e}-1 *(2 .-\right.$ $\left.\left.\left.1{ }^{*} \mathrm{r}\right)^{\wedge} 2\right)+0.6250000000 \mathrm{e}-2^{*}\left(2 .-1 .^{*} \mathrm{r}\right)^{\wedge} 2\right)-1{ }^{*} \mathrm{x}^{*} \mathrm{~h}^{\wedge} 2^{*}\left(0.9390024038 \mathrm{e}-5^{*}\left(-.2000000000 * \mathrm{r}^{\wedge} 2-\right.\right.$ $\left.0.3333333333 \mathrm{e}-1 *\left(2 .-1 .{ }^{*} \mathrm{r}\right)^{\wedge} 2\right)^{\wedge} 2+0.4340277778 \mathrm{e}-3^{*} \mathrm{r}^{*}\left(-.2000000000^{*} \mathrm{r}^{\wedge} 2-0.3333333333 \mathrm{e}-\right.$ $\left.\left.\left.1^{*}\left(2 .-1 *^{*} \mathrm{r}\right)^{\wedge} 2\right)+0.6250000000 \mathrm{e}-2^{*} \mathrm{r}^{\wedge} 2\right)\right)^{*}(1 .+\mathrm{h})^{*}\left(2 .-1{ }^{*} \mathrm{r}\right)+(0.3267973856 \mathrm{e}-$ $2 *\left((.1538461538 *(1 .+\mathrm{h}))^{*} \mathrm{~h}^{\wedge} 4^{*}\left(2 .-1{ }^{*} \mathrm{r}\right) *\left(-.2000000000 * \mathrm{r}^{\wedge} 2-0.3333333333 \mathrm{e}-1 *\left(2 .-1{ }^{*} \mathrm{r}\right)^{\wedge} 2\right)^{\wedge} 2-\right.$ $\left(.4444444444 *\left(-\left(1 *^{*}(1 .+\mathrm{h})\right)^{\wedge} \mathrm{h}^{*}\left(-.2000000000 * \mathrm{r}^{\wedge} 2-0.3333333333 \mathrm{e}-1 *\left(2 .-1{ }^{*} \mathrm{r}\right)^{\wedge} 2\right)-\right.\right.$ $\left.\left..2000000000^{*} \mathrm{~h}^{\wedge} 3^{*} \mathrm{r}^{\wedge} 2\right)\right)^{*} \mathrm{~h}^{\wedge} 3 * \mathrm{r}^{*}\left(-.2000000000 * \mathrm{r}^{\wedge} 2-0.3333333333 \mathrm{e}-1^{*}(2 .-\right.$ $\left.\left.\left.\left.1 *^{*} \mathrm{r}\right)^{\wedge} 2\right)\right)\right)^{*} \mathrm{x}^{\wedge} 18+\left(0.2164502165 \mathrm{e}-2^{*}\left(-\left(.1538461538^{*}\left(-\left(1 *^{*}(1 .+\mathrm{h})\right)^{*} \mathrm{~h}^{*}\left(-.2000000000^{*} \mathrm{r}^{\wedge} 2-\right.\right.\right.\right.\right.$ $\left.\left.\left.0.3333333333 \mathrm{e}-1^{*}\left(2 .-1 *^{*} \mathrm{r}\right)^{\wedge} 2\right)-.2000000000^{*} \mathrm{~h}^{\wedge} 3^{*} \mathrm{r}^{\wedge} 2\right)\right)^{*} \mathrm{~h}^{\wedge} 3^{*}\left(-.2000000000^{*} \mathrm{r}^{\wedge} 2-\right.$ $\left.0.3333333333 \mathrm{e}-1^{*}\left(2 .-1 .^{*} \mathrm{r}\right)^{\wedge} 2\right)^{\wedge} 2+0.4938271605 \mathrm{e}-1^{*} \mathrm{~h}^{\wedge} 6^{*} \mathrm{r}^{\wedge} 2^{*}\left(-.2000000000^{*} \mathrm{r}^{\wedge} 2-\right.$ $\left.\left.\left.0.3333333333 \mathrm{e}-1^{*}\left(2 .-1 *^{*} \mathrm{r}\right)^{\wedge} 2\right)^{\wedge} 2\right)\right)^{*} \mathrm{x}^{\wedge} 22+7.837560129^{*} 10^{\wedge}(-13)^{*} \mathrm{~h}^{\wedge} 6^{*} \mathrm{r}^{*}\left(-.2000000000^{*} \mathrm{r}^{\wedge} 2-\right.$ $\left.0.3333333333 \mathrm{e}-1^{*}\left(2 .-1{ }^{*} \mathrm{r}\right)^{\wedge} 2\right)^{\wedge} 3+\mathrm{x}^{*}\left(1.939051537^{*} 10^{\wedge}(-15)^{*} \mathrm{~h}^{\wedge} 6^{*}\left(-.2000000000^{*} \mathrm{r}^{\wedge} 2-\right.\right.$ $\left.0.3333333333 \mathrm{e}-1^{*}\left(2 .-1{ }^{*} \mathrm{r}\right)^{\wedge} 2\right)^{\wedge} 4+2.911093762^{*} 10^{\wedge}(-13)^{*} \mathrm{~h}^{\wedge} 6^{*}\left(2 .-1 *^{*} \mathrm{r}\right)^{*}\left(-.2000000000^{*} \mathrm{r}^{\wedge} 2-\right.$ $\left.0.3333333333 \mathrm{e}-1^{*}\left(2 .-1{ }^{*} \mathrm{r}\right)^{\wedge} 2\right)^{\wedge} 3-2.495223225^{*} 10^{\wedge}(-10)^{*}\left(-\left(1 *^{*}(1 .+\mathrm{h})\right)^{*} \mathrm{~h}^{*}\left(-.2000000000^{*} \mathrm{r}^{\wedge} 2-\right.\right.$ $\bar{u}_{1}(x, \alpha)=-\mathrm{h}^{*}\left(\mathrm{x}^{\wedge} 2^{*}(2-\mathrm{r})-(1 / 5)^{*} \mathrm{x}^{\wedge} 6^{*} \mathrm{r}^{\wedge} 2-(1 / 30)^{*} \mathrm{x}^{\wedge} 6^{*}(2-\mathrm{r})^{\wedge} 2\right)$

$\bar{u}_{2}(x, \alpha)=-(1+\mathrm{h})^{*} \mathrm{~h}^{*}\left(\mathrm{x}^{\wedge} 2^{*}(2-\mathrm{r})-(1 / 5)^{*} \mathrm{x}^{\wedge} 6^{*} \mathrm{r}^{\wedge} 2-(1 / 30)^{*} \mathrm{x}^{\wedge} 6^{*}(2-\mathrm{r})^{\wedge} 2\right)-\mathrm{h}^{*}\left(\mathrm{~h}^{\wedge} 2^{*}\left(0.9390024038 \mathrm{e}-5^{*}\left(-(1 / 5)^{*} \mathrm{r}^{\wedge} 2-\right.\right.\right.$ $\left.(1 / 30)^{*}(2-\mathrm{r})^{\wedge} 2\right)^{\wedge} 2+\left(0.4340277778 \mathrm{e}-3^{*}(2-\mathrm{r})\right)^{*}\left(-(1 / 5)^{*} \mathrm{r}^{\wedge} 2-(1 / 30)^{*}(2-\mathrm{r})^{\wedge} 2\right)+0.6250000000 \mathrm{e}-2^{*}(2-$ $\left.r)^{\wedge} 2\right)+x^{*} h^{\wedge} 2 *\left((1 / 13) *\left(-(1 / 5) * r^{\wedge} 2-(1 / 30) *(2-r)^{\wedge} 2\right)^{\wedge} 2 * x^{\wedge} 13+(2 / 9)^{*}{ }^{*}\left(-(1 / 5) * r^{\wedge} 2-(1 / 30) *(2-\right.\right.$ $\left.\left.\mathrm{r})^{\wedge} 2\right)^{*} \mathrm{x}^{\wedge} 9+(1 / 5)^{*} \mathrm{x}^{\wedge} 5^{*} \mathrm{r}^{\wedge} 2\right)-\mathrm{x}^{*} \mathrm{~h}^{\wedge} 2^{*}\left(0.9390024038 \mathrm{e}-5^{*}\left(-(1 / 5)^{*} \mathrm{r}^{\wedge} 2-(1 / 30)^{*}(2-\mathrm{r})^{\wedge} 2\right)^{\wedge} 2+0.4340277778 \mathrm{e}-3{ }^{*} \mathrm{r}^{*}(-\right.$ $\left.\left.\left.(1 / 5)^{*} \mathrm{r}^{\wedge} 2-(1 / 30)^{*}(2-\mathrm{r})^{\wedge} 2\right)+0.6250000000 \mathrm{e}-2^{*} \mathrm{r}^{\wedge} 2\right)\right)$

$\bar{u}_{3}(x, \alpha)=(1+\mathrm{h}) *\left(-(1+\mathrm{h}) * \mathrm{~h}^{*}\left(\mathrm{x}^{\wedge} 2^{*}(2-\mathrm{r})-(1 / 5)^{*} \mathrm{x}^{\wedge} 6^{*} \mathrm{r}^{\wedge} 2-(1 / 30) * \mathrm{x}^{\wedge} 6^{*}(2-\mathrm{r})^{\wedge} 2\right)-\right.$ $\mathrm{h}^{*}\left(\mathrm{~h}^{\wedge} 2^{*}\left(0.9390024038 \mathrm{e}-5^{*}\left(-(1 / 5)^{*} \mathrm{r}^{\wedge} 2-(1 / 30)^{*}(2-\mathrm{r})^{\wedge} 2\right)^{\wedge} 2+\left(0.4340277778 \mathrm{e}-3^{*}(2-\mathrm{r})\right)^{*}(-\right.\right.$ $\left.\left.(1 / 5) * r^{\wedge} 2-(1 / 30) *(2-r)^{\wedge} 2\right)+0.6250000000 \mathrm{e}-2^{*}(2-r)^{\wedge} 2\right)+x^{*} h^{\wedge} 2^{*}\left((1 / 13) *\left(-(1 / 5)^{*} r^{\wedge} 2-\right.\right.$ $\left.\left.(1 / 30)^{*}(2-r)^{\wedge} 2\right)^{\wedge} 2^{*} x^{\wedge} 13+(2 / 9)^{*} r^{*}\left(-(1 / 5)^{*} r^{\wedge} 2-(1 / 30)^{*}(2-r)^{\wedge} 2\right)^{*} x^{\wedge} 9+(1 / 5)^{*} x^{\wedge} 5^{*} r^{\wedge} 2\right)-$ 
$\mathrm{x}^{*} \mathrm{~h}^{\wedge} 2^{*}\left(0.9390024038 \mathrm{e}-5^{*}\left(-(1 / 5) * \mathrm{r}^{\wedge} 2-(1 / 30) *(2-\mathrm{r})^{\wedge} 2\right)^{\wedge} 2+0.4340277778 \mathrm{e}-3^{*} \mathrm{r}^{*}\left(-(1 / 5) * \mathrm{r}^{\wedge} 2-\right.\right.$ $\left.\left.\left.\left.(1 / 30) *(2-r)^{\wedge} 2\right)+0.6250000000 \mathrm{e}-2^{*} \mathrm{r}^{\wedge} 2\right)\right)\right)-\mathrm{h} *\left(0.6801333060 \mathrm{e}-5^{*} \mathrm{~h}^{\wedge} 6^{*}\left(-.2000000000^{*} \mathrm{r}^{\wedge} 2-\right.\right.$ $\left.0.3333333333 \mathrm{e}-1^{*}\left(2 .-1{ }^{*} \mathrm{r}\right)^{\wedge} 2\right)^{\wedge} 4^{*} \mathrm{t}^{\wedge} 30+0.5259697569 \mathrm{e}-4^{*} \mathrm{~h}^{\wedge} 6^{*} \mathrm{r}^{*}\left(-.2000000000^{*} \mathrm{r}^{\wedge} 2-\right.$ $\left.0.3333333333 \mathrm{e}-1^{*}\left(2 .-1 *^{*} \mathrm{r}\right)^{\wedge} 2\right)^{\wedge} 3^{*} \mathrm{t}^{\wedge} 26+\left(0.2164502165 \mathrm{e}-2^{*}\left(-\left(.1538461538 *\left(-(1 . *(1 .+\mathrm{h}))^{*} \mathrm{~h}^{*}(-\right.\right.\right.\right.$ $\left.\left.\left..2000000000^{*} r^{\wedge} 2-0.3333333333 \mathrm{e}-1^{*}\left(2 .-1{ }^{*} \mathrm{r}\right)^{\wedge} 2\right)-.2000000000^{*} \mathrm{~h}^{\wedge} 3^{*} \mathrm{r}^{\wedge} 2\right)\right)^{*} \mathrm{~h}^{\wedge} 3^{*}(-$ $\left..2000000000 * \mathrm{r}^{\wedge} 2-0.3333333333 \mathrm{e}-1 *\left(2 .-1{ }^{*} \mathrm{r}\right)^{\wedge} 2\right)^{\wedge} 2+0.4938271605 \mathrm{e}-1^{*} \mathrm{~h}^{\wedge} 6^{*} \mathrm{r}^{\wedge} 2^{*}(-$ $\left.\left.\left..2000000000 * r^{\wedge} 2-0.3333333333 \mathrm{e}-1^{*}\left(2 .-1 *^{*} \mathrm{r}\right)^{\wedge} 2\right)^{\wedge} 2\right)\right)^{*} \mathrm{t}^{\wedge} 22+(0.3267973856 \mathrm{e}-$ $2^{*}\left((.1538461538 *(1 .+\mathrm{h}))^{*} \mathrm{~h}^{\wedge} 4^{*}\left(2 .-1{ }^{*} \mathrm{r}\right)^{*}\left(-.2000000000^{*} \mathrm{r}^{\wedge} 2-0.3333333333 \mathrm{e}-1^{*}\left(2 .-1{ }^{*} \mathrm{r}\right)^{\wedge} 2\right)^{\wedge} 2-\right.$ $\left(.4444444444^{*}\left(-\left(1 *^{*}(1 .+\mathrm{h})\right)^{*} \mathrm{~h}^{*}\left(-.2000000000^{*} \mathrm{r}^{\wedge} 2-0.3333333333 \mathrm{e}-1^{*}\left(2 .-1 .^{*} \mathrm{r}\right)^{\wedge} 2\right)-\right.\right.$ $\left.\left..2000000000^{*} \mathrm{~h}^{\wedge} 3^{*} \mathrm{r}^{\wedge} 2\right)\right)^{*} \mathrm{~h}^{\wedge} 3^{*} \mathrm{r}^{*}\left(-.2000000000 * \mathrm{r}^{\wedge} 2-0.3333333333 \mathrm{e}-1^{*}(2 .-\right.$

$\left.\left.\left.\left.1 *^{*} \mathrm{r}\right)^{\wedge} 2\right)\right)\right)^{*} \mathrm{t}^{\wedge} 18+0.6410256412 \mathrm{e}-3^{*} \mathrm{~h}^{\wedge} 4^{*}\left(\mathrm{~h}^{\wedge} 2^{*}\left(0.9390024038 \mathrm{e}-5^{*}\left(-.2000000000 * \mathrm{r}^{\wedge} 2-\right.\right.\right.$ $\left.0.3333333333 \mathrm{e}-1^{*}\left(2 .-1{ }^{*} \mathrm{r}\right)^{\wedge} 2\right)^{\wedge} 2+\left(0.4340277778 \mathrm{e}-3^{*}\left(2 .-1 *^{*} \mathrm{r}\right)\right)^{*}\left(-.2000000000^{*} \mathrm{r}^{\wedge} 2-\right.$ $\left.\left.0.3333333333 \mathrm{e}-1^{*}\left(2 .-1 *^{*} \mathrm{r}\right)^{\wedge} 2\right)+0.6250000000 \mathrm{e}-2^{*}\left(2 .-1{ }^{*} \mathrm{r}\right)^{\wedge} 2\right)-1{ }^{*} \mathrm{x}^{*} \mathrm{~h}^{\wedge} 2^{*}\left(0.9390024038 \mathrm{e}-5^{*}(-\right.$ $\left..2000000000^{*} r^{\wedge} 2-0.3333333333 \mathrm{e}-1 *\left(2 .-1{ }^{*} \mathrm{r}\right)^{\wedge} 2\right)^{\wedge} 2+0.4340277778 \mathrm{e}-3^{*} \mathrm{r}^{*}\left(-.2000000000^{*} \mathrm{r}^{\wedge} 2-\right.$ $\left.\left.\left.0.3333333333 \mathrm{e}-1^{*}\left(2 .-1 *^{*} \mathrm{r}\right)^{\wedge} 2\right)+0.6250000000 \mathrm{e}-2^{*} \mathrm{r}^{\wedge} 2\right)\right)^{*}\left(-.2000000000 * \mathrm{r}^{\wedge} 2-0.3333333333 \mathrm{e}-\right.$ $\left.1^{*}\left(2 .-1{ }^{*} \mathrm{r}\right)^{\wedge} 2\right)^{\wedge} 2^{*} \mathrm{t}^{\wedge} 16+\left(0.5494505494 \mathrm{e}-2^{*}\left(\left(.4444444444^{*}(1 .+\mathrm{h})\right)^{*} \mathrm{~h}^{\wedge} 4^{*}\left(2 .-1 .^{*} \mathrm{r}\right)^{*} \mathrm{r}^{*}(-\right.\right.$ $\left..2000000000^{*} \mathrm{r}^{\wedge} 2-0.3333333333 \mathrm{e}-1^{*}\left(2 .-1 .^{*} \mathrm{r}\right)^{\wedge} 2\right)+\left(-\left(1 *^{*}(1 .+\mathrm{h})\right)^{*} \mathrm{~h}^{*}\left(-.2000000000^{*} \mathrm{r}^{\wedge} 2-\right.\right.$ $\left.\left.\left.\left.0.3333333333 \mathrm{e}-1^{*}\left(2 .-1 .^{*} \mathrm{r}\right)^{\wedge} 2\right)-.2000000000^{*} \mathrm{~h}^{\wedge} 3^{*} \mathrm{r}^{\wedge} 2\right)^{\wedge} 2\right)\right)^{*} \mathrm{t}^{\wedge} 14+0.3367003367 \mathrm{e}-$ $2^{*} \mathrm{~h}^{\wedge} 4^{*}\left(\mathrm{~h}^{\wedge} 2^{*}\left(0.9390024038 \mathrm{e}-5^{*}\left(-.2000000000^{*} \mathrm{r}^{\wedge} 2-0.3333333333 \mathrm{e}-1^{*}(2 .-\right.\right.\right.$ $\left.\left.1{ }^{*} \mathrm{r}\right)^{\wedge} 2\right)^{\wedge} 2+\left(0.4340277778 \mathrm{e}-3^{*}\left(2 .-1{ }^{*} \mathrm{r}\right)\right)^{*}\left(-.2000000000^{*} \mathrm{r}^{\wedge} 2-0.3333333333 \mathrm{e}-1^{*}(2 .-\right.$ $\left.\left.\left.1{ }^{*} \mathrm{r}\right)^{\wedge} 2\right)+0.6250000000 \mathrm{e}-2^{*}\left(2 .-1{ }^{*} \mathrm{r}\right)^{\wedge} 2\right)-1{ }^{*} \mathrm{x}^{*} \mathrm{~h}^{\wedge} 2^{*}\left(0.9390024038 \mathrm{e}-5^{*}\left(-.2000000000^{*} \mathrm{r}^{\wedge} 2-\right.\right.$ $\left.0.3333333333 \mathrm{e}-1^{*}\left(2 .-1{ }^{*} \mathrm{r}\right)^{\wedge} 2\right)^{\wedge} 2+0.4340277778 \mathrm{e}-3^{*} \mathrm{r}^{*}\left(-.2000000000^{*} \mathrm{r}^{\wedge} 2-0.3333333333 \mathrm{e}-\right.$ $\left.\left.\left.1 *\left(2 .-1{ }^{*} \mathrm{r}\right)^{\wedge} 2\right)+0.6250000000 \mathrm{e}-2^{*} \mathrm{r}^{\wedge} 2\right)\right)^{*} \mathrm{r}^{*}\left(-.2000000000 * \mathrm{r}^{\wedge} 2-0.3333333333 \mathrm{e}-1 *(2 .-\right.$ $\left.\left.1{ }^{*} \mathrm{r}\right)^{\wedge} 2\right)^{*} \mathrm{t}^{\wedge} 12-\left(0.2222222222 \mathrm{e}-1^{*}(1 .+\mathrm{h})\right)^{*} \mathrm{~h}^{*}\left(2 .-1{ }^{*} \mathrm{r}\right)^{*}\left(-\left(1{ }^{*}(1 .+\mathrm{h})\right)^{*} \mathrm{~h}^{*}\left(-.2000000000^{*} \mathrm{r}^{\wedge} 2-\right.\right.$ $\left.\left.0.3333333333 \mathrm{e}-1^{*}\left(2 .-1{ }^{*} \mathrm{r}\right)^{\wedge} 2\right)-.2000000000^{*} \mathrm{~h}^{\wedge} 3^{*} \mathrm{r}^{\wedge} 2\right)^{*} \mathrm{t}^{\wedge} 10-0.3571428571 \mathrm{e}-$ $1^{*} \mathrm{~h}^{*}\left(\mathrm{~h}^{\wedge} 2^{*}\left(0.9390024038 \mathrm{e}-5^{*}\left(-.2000000000^{*} \mathrm{r}^{\wedge} 2-0.3333333333 \mathrm{e}-1^{*}(2 .-\right.\right.\right.$ $\left.\left.1 *^{*} \mathrm{r}\right)^{\wedge} 2\right)^{\wedge} 2+\left(0.4340277778 \mathrm{e}-3^{*}\left(2 .-1{ }^{*} \mathrm{r}\right)\right)^{*}\left(-.2000000000^{*} \mathrm{r}^{\wedge} 2-0.3333333333 \mathrm{e}-1^{*}(2 .-\right.$ $\left.\left.1 . * r)^{\wedge} 2\right)+0.6250000000 \mathrm{e}-2^{*}\left(2 .-1{ }^{*} \mathrm{r}\right)^{\wedge} 2\right)-1{ }^{*} \mathrm{x}^{*} \mathrm{~h}^{\wedge} 2^{*}\left(0.9390024038 \mathrm{e}-5^{*}\left(-.2000000000^{*} \mathrm{r}^{\wedge} 2-\right.\right.$ $\left.0.3333333333 \mathrm{e}-1^{*}\left(2 .-1{ }^{*} \mathrm{r}\right)^{\wedge} 2\right)^{\wedge} 2+0.4340277778 \mathrm{e}-3^{*} \mathrm{r}^{*}\left(-.2000000000^{*} \mathrm{r}^{\wedge} 2-0.3333333333 \mathrm{e}-\right.$ $\left.\left.\left.1^{*}\left(2 .-1 *^{*} \mathrm{r}\right)^{\wedge} 2\right)+0.6250000000 \mathrm{e}-2^{*} \mathrm{r}^{\wedge} 2\right)\right)^{*}\left(-(1 *(1 .+\mathrm{h}))^{*} \mathrm{~h}^{*}\left(-.2000000000^{*} \mathrm{r}^{\wedge} 2-0.3333333333 \mathrm{e}-\right.\right.$ $\left.\left.1^{*}\left(2 .-1{ }^{*} \mathrm{r}\right)^{\wedge} 2\right)-.2000000000^{*} \mathrm{~h}^{\wedge} 3^{*} \mathrm{r}^{\wedge} 2\right)^{*} \mathrm{t}^{\wedge} 8+0.3333333333 \mathrm{e}-1^{*}(1 .+\mathrm{h})^{\wedge} 2^{*} \mathrm{~h}^{\wedge} 2^{*}(2 .-$

$\left.1{ }^{*} \mathrm{r}\right)^{\wedge} 2^{*} \mathrm{t}^{\wedge} 6+.1666666667 * \mathrm{~h}^{\wedge} 2^{*}\left(\mathrm{~h}^{\wedge} 2^{*}\left(0.9390024038 \mathrm{e}-5^{*}\left(-.2000000000^{*} \mathrm{r}^{\wedge} 2-0.3333333333 \mathrm{e}-\right.\right.\right.$ $\left.1^{*}\left(2 .-1 *^{*} \mathrm{r}\right)^{\wedge} 2\right)^{\wedge} 2+\left(0.4340277778 \mathrm{e}-3^{*}\left(2 .-1{ }^{*} \mathrm{r}\right)\right)^{*}\left(-.2000000000^{*} \mathrm{r}^{\wedge} 2-0.3333333333 \mathrm{e}-1^{*}(2 .-\right.$ $\left.\left.1 . * r)^{\wedge} 2\right)+0.6250000000 \mathrm{e}-2^{*}\left(2 .-1{ }^{*} \mathrm{r}\right)^{\wedge} 2\right)-1{ }^{*} \mathrm{x}^{*} \mathrm{~h}^{\wedge} 2^{*}\left(0.9390024038 \mathrm{e}-5^{*}\left(-.2000000000^{*} \mathrm{r}^{\wedge} 2-\right.\right.$ $\left.0.3333333333 \mathrm{e}-1^{*}\left(2 .-1 .{ }^{*} \mathrm{r}\right)^{\wedge} 2\right)^{\wedge} 2+0.4340277778 \mathrm{e}-3^{*} \mathrm{r}^{*}\left(-.2000000000^{*} \mathrm{r}^{\wedge} 2-0.3333333333 \mathrm{e}-\right.$ $\left.\left.\left.1 *\left(2 .-1{ }^{*} \mathrm{r}\right)^{\wedge} 2\right)+0.6250000000 \mathrm{e}-2 * \mathrm{r}^{\wedge} 2\right)\right)^{*}(1 .+\mathrm{h}) *(2 .-$

$\bar{U}(x, \alpha) \quad=-h^{*}\left(\mathrm{x}^{\wedge} 2^{*}(2-\mathrm{r})-(1 / 5)^{*} \mathrm{x}^{\wedge} 6^{*} \mathrm{r}^{\wedge} 2-(1 / 30)^{*} \mathrm{x}^{\wedge} 6^{*}(2-\mathrm{r})^{\wedge} 2\right)-(1+\mathrm{h})^{*} \mathrm{~h}^{*}\left(\mathrm{x}^{\wedge} 2^{*}(2-\mathrm{r})-\right.$ $\left.(1 / 5)^{*} \mathrm{x}^{\wedge} 6^{*} \mathrm{r}^{\wedge} 2-(1 / 30) * \mathrm{x}^{\wedge} 6^{*}(2-\mathrm{r})^{\wedge} 2\right)+\mathrm{h}^{*}\left(\mathrm{~h}^{\wedge} 2^{*}\left(0.9390024038 \mathrm{e}-5^{*}\left((1 / 5) * \mathrm{r}^{\wedge} 2-(1 / 30) *(2-\right.\right.\right.$

$\left.r)^{\wedge} 2\right)^{\wedge} 2+\left(0.4340277778 \mathrm{e}-3^{*}(2-\mathrm{r})\right)^{*}\left((1 / 5)^{*} \mathrm{r}^{\wedge} 2-(1 / 30)^{*}(2-\mathrm{r})^{\wedge} 2\right)+0.6250000000 \mathrm{e}-2^{*}(2-$ $\left.r)^{\wedge} 2\right)+x^{*} h^{\wedge} 2^{*}\left((1 / 13) *\left((1 / 5)^{*} r^{\wedge} 2-(1 / 30) *(2-r)^{\wedge} 2\right)^{\wedge} 2^{*} x^{\wedge} 13+(2 / 9) * r^{*}\left((1 / 5) * r^{\wedge} 2-(1 / 30) *(2-\right.\right.$ $\left.\left.r)^{\wedge} 2\right)^{*} x^{\wedge} 9+(1 / 5)^{*} x^{\wedge} 5^{*} r^{\wedge} 2\right)+x^{*} h^{\wedge} 2^{*}\left(0.9390024038 e-5^{*}\left((1 / 5)^{*} r^{\wedge} 2-(1 / 30) *(2-\right.\right.$

$\left.\left.\left.r)^{\wedge} 2\right)^{\wedge} 2+0.4340277778 \mathrm{e}-3^{*} \mathrm{r}^{*}\left((1 / 5)^{*} \mathrm{r}^{\wedge} 2-(1 / 30)^{*}(2-\mathrm{r})^{\wedge} 2\right)+0.6250000000 \mathrm{e}-2^{*} \mathrm{r}^{\wedge} 2\right)\right)+(1+\mathrm{h})^{*}(-$ $(1+h)^{*} h^{*}\left(x^{\wedge} 2^{*}(2-r)-(1 / 5)^{*} x^{\wedge} 6^{*} r^{\wedge} 2-(1 / 30)^{*} x^{\wedge} 6^{*}(2-r)^{\wedge} 2\right)+h^{*}\left(h^{\wedge} 2^{*}(0.9390024038 \mathrm{e}-\right.$ $5^{*}\left((1 / 5) * r^{\wedge} 2-(1 / 30) *(2-r)^{\wedge} 2\right)^{\wedge} 2+\left(0.4340277778 \mathrm{e}-3^{*}(2-r)\right)^{*}\left(-(1 / 5) * r^{\wedge} 2-(1 / 30) *(2-\right.$ $\left.\left.r)^{\wedge} 2\right)+0.6250000000 \mathrm{e}-2^{*}(2-\mathrm{r})^{\wedge} 2\right)+\mathrm{x}^{*} \mathrm{~h}^{\wedge} 2^{*}\left((1 / 13) *\left(-(1 / 5)^{*} \mathrm{r}^{\wedge} 2-(1 / 30)^{*}(2-\right.\right.$ $\left.r)^{\wedge} 2\right)^{\wedge} 2^{*} x^{\wedge} 13+(2 / 9) * r^{*}\left(-(1 / 5) * r^{\wedge} 2-(1 / 30) *(2-\right.$ $\left.\left.r)^{\wedge} 2\right)^{*} x^{\wedge} 9+(1 / 5) * x^{\wedge} 5^{*} r^{\wedge} 2\right)+x^{*} h^{\wedge} 2^{*}\left(0.9390024038 e-5 *\left(-(1 / 5) * r^{\wedge} 2-(1 / 30) *(2-\right.\right.$ $\left.\mathrm{r})^{\wedge} 2\right)^{\wedge} 2+0.4340277778 \mathrm{e}-3^{*} \mathrm{r}^{*}\left(-(1 / 5)^{*} \mathrm{r}^{\wedge} 2-(1 / 30)^{*}(2-\mathrm{r})^{\wedge} 2\right)+0.6250000000 \mathrm{e}-$ $\left.\left.\left.2^{*} \mathrm{r}^{\wedge} 2\right)\right)\right)+\mathrm{h}^{*}\left(0.6801333060 \mathrm{e}-5^{*} \mathrm{~h}^{\wedge} 6^{*}\left(-.2000000000 * \mathrm{r}^{\wedge} 2-0.3333333333 \mathrm{e}-1^{*}(2 .-\right.\right.$ 
1. $\left.\left.{ }^{*}\right)^{\wedge} 2\right)^{\wedge} 4 * \mathrm{x}^{\wedge} 30+0.5259697569 \mathrm{e}-4 * \mathrm{~h}^{\wedge} 6 * \mathrm{r}^{*}\left(-.2000000000^{*} \mathrm{r}^{\wedge} 2-0.3333333333 \mathrm{e}-1 *(2 .-\right.$ $\left.\left.1 *^{*} \mathrm{r}\right)^{\wedge} 2\right)^{\wedge} 3^{*} \mathrm{x}^{\wedge} 26+\left(0.2164502165 \mathrm{e}-2^{*}\left(-\left(.1538461538 *\left(-\left(1 *^{*}(1 .+\mathrm{h})\right)^{*} \mathrm{~h}^{*}\left(-.2000000000^{*} \mathrm{r}^{\wedge} 2-\right.\right.\right.\right.\right.$ $\left.\left.\left.0.3333333333 \mathrm{e}-1^{*}\left(2 .-1{ }^{*} \mathrm{r}\right)^{\wedge} 2\right)-.2000000000^{*} \mathrm{~h}^{\wedge} 3^{*} \mathrm{r}^{\wedge} 2\right)\right)^{*} \mathrm{~h}^{\wedge} 3^{*}\left(-.2000000000^{*} \mathrm{r}^{\wedge} 2-\right.$ $\left.0.3333333333 \mathrm{e}-1 *\left(2 .-1 *^{*} \mathrm{r}\right)^{\wedge} 2\right)^{\wedge} 2+0.4938271605 \mathrm{e}-1 * \mathrm{~h}^{\wedge} 6^{*} \mathrm{r}^{\wedge} 2^{*}\left(-.2000000000 * \mathrm{r}^{\wedge} 2-\right.$ $\left.\left.\left.0.3333333333 \mathrm{e}-1^{*}\left(2 .-1 *^{*} \mathrm{r}\right)^{\wedge} 2\right)^{\wedge} 2\right)\right)^{*} \mathrm{x}^{\wedge} 22+\left(0.3267973856 \mathrm{e}-2^{*}\left((.1538461538 *(1 .+\mathrm{h}))^{*} \mathrm{~h}^{\wedge} 4^{*}(2 .-\right.\right.$ $\left.1{ }^{*} \mathrm{r}\right) *\left(-.2000000000^{*} \mathrm{r}^{\wedge} 2-0.3333333333 \mathrm{e}-1^{*}\left(2 .-1{ }^{*} \mathrm{r}\right)^{\wedge} 2\right)^{\wedge} 2-\left(.4444444444^{*}\left(-\left(1 *^{*}(1 .+\mathrm{h})\right)^{*} \mathrm{~h}^{*}(-\right.\right.$ $\left.\left.\left..2000000000^{*} \mathrm{r}^{\wedge} 2-0.3333333333 \mathrm{e}-1^{*}\left(2 .-1 *^{*} \mathrm{r}\right)^{\wedge} 2\right)-.2000000000^{*} \mathrm{~h}^{\wedge} 3^{*} \mathrm{r}^{\wedge} 2\right)\right)^{*} \mathrm{~h}^{\wedge} 3^{*} \mathrm{r}^{*}(-$ $\left.\left.\left..2000000000^{*} \mathrm{r}^{\wedge} 2-0.3333333333 \mathrm{e}-1^{*}\left(2 .-1 *^{*} \mathrm{r}\right)^{\wedge} 2\right)\right)\right)^{*} \mathrm{x}^{\wedge} 18+0.6410256412 \mathrm{e}-$ $3^{*} \mathrm{~h}^{\wedge} 4^{*}\left(\mathrm{~h}^{\wedge} 2^{*}\left(0.9390024038 \mathrm{e}-5^{*}\left(-.2000000000 * \mathrm{r}^{\wedge} 2-0.3333333333 \mathrm{e}-1^{*}(2 .-\right.\right.\right.$ $\left.\left.1{ }^{*} \mathrm{r}\right)^{\wedge} 2\right)^{\wedge} 2+\left(0.4340277778 \mathrm{e}-3^{*}\left(2 .-1{ }^{*} \mathrm{r}\right)\right)^{*}\left(-.2000000000^{*} \mathrm{r}^{\wedge} 2-0.3333333333 \mathrm{e}-1 *(2 .-\right.$ $\left.\left.\left.1 .{ }^{*} \mathrm{r}\right)^{\wedge} 2\right)+0.6250000000 \mathrm{e}-2^{*}\left(2 .-1{ }^{*} \mathrm{r}\right)^{\wedge} 2\right)-1{ }^{*} \mathrm{x}^{*} \mathrm{~h}^{\wedge} 2^{*}\left(0.9390024038 \mathrm{e}-5^{*}\left(-.2000000000 * \mathrm{r}^{\wedge} 2-\right.\right.$ $\left.0.3333333333 \mathrm{e}-1^{*}\left(2 .-1{ }^{*} \mathrm{r}\right)^{\wedge} 2\right)^{\wedge} 2+0.4340277778 \mathrm{e}-3^{*} \mathrm{r}^{*}\left(-.2000000000^{*} \mathrm{r}^{\wedge} 2-0.3333333333 \mathrm{e}-\right.$ $\left.\left.\left.1^{*}\left(2 .-1 *^{*} \mathrm{r}\right)^{\wedge} 2\right)+0.6250000000 \mathrm{e}-2^{*} \mathrm{r}^{\wedge} 2\right)\right)^{*}\left(-.2000000000^{*} \mathrm{r}^{\wedge} 2-0.3333333333 \mathrm{e}-1^{*}(2 .-\right.$ $\left.1 . * \mathrm{r})^{\wedge} 2\right)^{\wedge} 2 * \mathrm{x}^{\wedge} 16+\left(0.5494505494 \mathrm{e}-2^{*}\left((.4444444444 *(1 .+\mathrm{h}))^{*} \mathrm{~h}^{\wedge} 4^{*}\left(2 .-1{ }^{*} \mathrm{r}\right) * \mathrm{r}^{*}(-\right.\right.$ $\left..2000000000^{*} \mathrm{r}^{\wedge} 2-0.3333333333 \mathrm{e}-1^{*}\left(2 .-1 *^{*} \mathrm{r}\right)^{\wedge} 2\right)+\left(-\left(1 *^{*}(1 .+\mathrm{h})\right)^{*} \mathrm{~h}^{*}\left(-.2000000000^{*} \mathrm{r}^{\wedge} 2-\right.\right.$ $\left.\left.\left.\left.0.3333333333 \mathrm{e}-1^{*}\left(2 .-1{ }^{*} \mathrm{r}\right)^{\wedge} 2\right)-.2000000000^{*} \mathrm{~h}^{\wedge} 3^{*} \mathrm{r}^{\wedge} 2\right)^{\wedge} 2\right)\right)^{*} \mathrm{x}^{\wedge} 14+0.3367003367 \mathrm{e}-$ $2^{*} \mathrm{~h}^{\wedge} 4^{*}\left(\mathrm{~h}^{\wedge} 2^{*}\left(0.9390024038 \mathrm{e}-5^{*}\left(-.2000000000^{*} \mathrm{r}^{\wedge} 2-0.3333333333 \mathrm{e}-1^{*}(2 .-\right.\right.\right.$

$\left.\left.1{ }^{*} \mathrm{r}\right)^{\wedge} 2\right)^{\wedge} 2+\left(0.4340277778 \mathrm{e}-3^{*}\left(2 .-1{ }^{*} \mathrm{r}\right)\right)^{*}\left(-.2000000000^{*} \mathrm{r}^{\wedge} 2-0.3333333333 \mathrm{e}-1 *(2 .-\right.$ $\left.\left.\left.1{ }^{*} \mathrm{r}\right)^{\wedge} 2\right)+0.6250000000 \mathrm{e}-2^{*}\left(2 .-1{ }^{*} \mathrm{r}\right)^{\wedge} 2\right)-1{ }^{*} \mathrm{x}^{*} \mathrm{~h}^{\wedge} 2^{*}\left(0.9390024038 \mathrm{e}-5^{*}\left(-.2000000000^{*} \mathrm{r}^{\wedge} 2-\right.\right.$ $\left.0.3333333333 \mathrm{e}-1^{*}\left(2 .-1{ }^{*} \mathrm{r}\right)^{\wedge} 2\right)^{\wedge} 2+0.4340277778 \mathrm{e}-3^{*} \mathrm{r}^{*}\left(-.2000000000^{*} \mathrm{r}^{\wedge} 2-0.3333333333 \mathrm{e}-\right.$ $\left.\left.\left.1 *\left(2 .-1{ }^{*} \mathrm{r}\right)^{\wedge} 2\right)+0.6250000000 \mathrm{e}-2^{*} \mathrm{r}^{\wedge} 2\right)\right)^{*} \mathrm{r}^{*}\left(-.2000000000 * \mathrm{r}^{\wedge} 2-0.3333333333 \mathrm{e}-1^{*}(2 .-\right.$ $\left.\left.1{ }^{*} \mathrm{r}\right)^{\wedge} 2\right)^{*} \mathrm{x}^{\wedge} 12-\left(0.2222222222 \mathrm{e}-1^{*}(1 .+\mathrm{h})\right)^{*} \mathrm{~h}^{*}\left(2 .-1{ }^{*} \mathrm{r}\right)^{*}\left(-\left(1 *^{*}(1 .+\mathrm{h})\right)^{*} \mathrm{~h}^{*}\left(-.2000000000^{*} \mathrm{r}^{\wedge} 2-\right.\right.$ $\left.\left.0.3333333333 \mathrm{e}-1^{*}\left(2 .-1{ }^{*} \mathrm{r}\right)^{\wedge} 2\right)-.2000000000^{*} \mathrm{~h}^{\wedge} 3^{*} \mathrm{r}^{\wedge} 2\right)^{*} \mathrm{x}^{\wedge} 10-0.3571428571 \mathrm{e}-$ $1^{*} \mathrm{~h}^{*}\left(\mathrm{~h}^{\wedge} 2^{*}\left(0.9390024038 \mathrm{e}-5^{*}\left(-.2000000000^{*} \mathrm{r}^{\wedge} 2-0.3333333333 \mathrm{e}-1^{*}(2 .-\right.\right.\right.$ $\left.\left.1{ }^{*} \mathrm{r}\right)^{\wedge} 2\right)^{\wedge} 2+\left(0.4340277778 \mathrm{e}-3^{*}\left(2 .-1{ }^{*} \mathrm{r}\right)\right)^{*}\left(-.2000000000^{*} \mathrm{r}^{\wedge} 2-0.3333333333 \mathrm{e}-1 *(2 .-\right.$ $\left.\left.\left.1 *^{*} \mathrm{r}\right)^{\wedge} 2\right)+0.6250000000 \mathrm{e}-2^{*}\left(2 .-1{ }^{*} \mathrm{r}\right)^{\wedge} 2\right)-1{ }^{*} \mathrm{x}^{*} \mathrm{~h}^{\wedge} 2^{*}\left(0.9390024038 \mathrm{e}-5^{*}\left(-.2000000000^{*} \mathrm{r}^{\wedge} 2-\right.\right.$ $\left.0.3333333333 \mathrm{e}-1^{*}\left(2 .-1 .^{*} \mathrm{r}\right)^{\wedge} 2\right)^{\wedge} 2+0.4340277778 \mathrm{e}-3^{*} \mathrm{r}^{*}\left(-.2000000000^{*} \mathrm{r}^{\wedge} 2-0.3333333333 \mathrm{e}-\right.$ $\left.\left.\left.1^{*}\left(2 .-1{ }^{*} \mathrm{r}\right)^{\wedge} 2\right)+0.6250000000 \mathrm{e}-2^{*} \mathrm{r}^{\wedge} 2\right)\right)^{*}\left(-\left(1 .^{*}(1 .+\mathrm{h})\right)^{*} \mathrm{~h}^{*}\left(-.2000000000^{*} \mathrm{r}^{\wedge} 2-0.3333333333 \mathrm{e}-\right.\right.$ $\left.\left.1 *\left(2 .-1{ }^{*} \mathrm{r}\right)^{\wedge} 2\right)-.2000000000^{*} \mathrm{~h}^{\wedge} 3^{*} \mathrm{r}^{\wedge} 2\right)^{*} \mathrm{x}^{\wedge} 8+0.3333333333 \mathrm{e}-1 *(1 .+\mathrm{h})^{\wedge} 2^{*} \mathrm{~h}^{\wedge} 2^{*}(2 .-$

$\left.1{ }^{*} \mathrm{r}\right)^{\wedge} 2^{*} \mathrm{x}^{\wedge} 6+.1666666667 * \mathrm{~h}^{\wedge} 2^{*}\left(\mathrm{~h}^{\wedge} 2^{*}\left(0.9390024038 \mathrm{e}-5^{*}\left(-.2000000000^{*} \mathrm{r}^{\wedge} 2-0.3333333333 \mathrm{e}-\right.\right.\right.$ $\left.1^{*}\left(2 .-1{ }^{*} \mathrm{r}\right)^{\wedge} 2\right)^{\wedge} 2+\left(0.4340277778 \mathrm{e}-3^{*}\left(2 .-1{ }^{*} \mathrm{r}\right)\right)^{*}\left(-.2000000000^{*} \mathrm{r}^{\wedge} 2-0.3333333333 \mathrm{e}-1^{*}(2 .-\right.$ $\left.\left.1 . * r)^{\wedge} 2\right)+0.6250000000 \mathrm{e}-2^{*}\left(2 .-1{ }^{*} \mathrm{r}\right)^{\wedge} 2\right)-1{ }^{*} \mathrm{x}^{*} \mathrm{~h}^{\wedge} 2^{*}\left(0.9390024038 \mathrm{e}-5^{*}\left(-.2000000000^{*} \mathrm{r}^{\wedge} 2-\right.\right.$ $\left.0.3333333333 \mathrm{e}-1 *\left(2 .-1 *^{*} \mathrm{r}\right)^{\wedge} 2\right)^{\wedge} 2+0.4340277778 \mathrm{e}-3^{*} \mathrm{r}^{*}\left(-.2000000000^{*} \mathrm{r}^{\wedge} 2-0.3333333333 \mathrm{e}-\right.$ $\left.\left.\left.1 *\left(2 .-1 *^{*} \mathrm{r}\right)^{\wedge} 2\right)+0.6250000000 \mathrm{e}-2^{*} \mathrm{r}^{\wedge} 2\right)\right)^{*}(1 .+\mathrm{h}) *(2 .-$

$1 . * \mathrm{r}) * \mathrm{x}^{\wedge} 4+.5000000000 * \mathrm{~h}^{\wedge} 2^{*}\left(\mathrm{~h}^{\wedge} 2^{*}\left(0.9390024038 \mathrm{e}-5^{*}\left(-.2000000000 * \mathrm{r}^{\wedge} 2-0.3333333333 \mathrm{e}-\right.\right.\right.$

Fig(1a)Exact solution $\underline{u}(x, \alpha)$

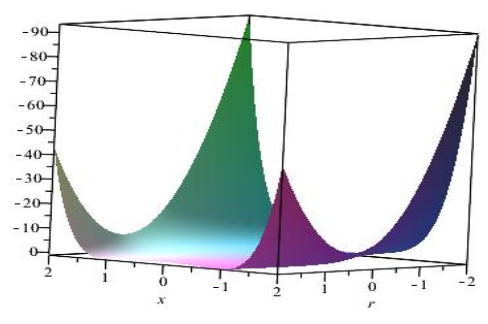

Fig(2a)Exact solution $\bar{u}(x, \alpha) \quad$ Fig $(2 \mathrm{~b})$ Approximate solution $\bar{u}(x, \alpha)$

Fig(1b) Approximate solution $u(x, \alpha)$

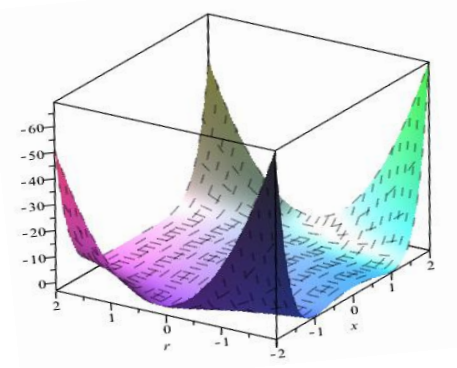

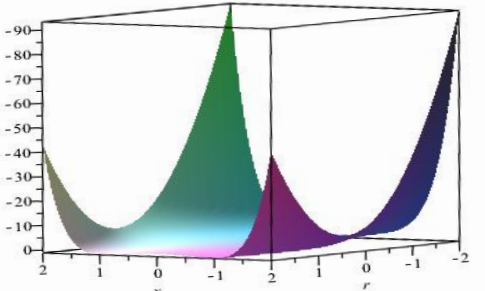

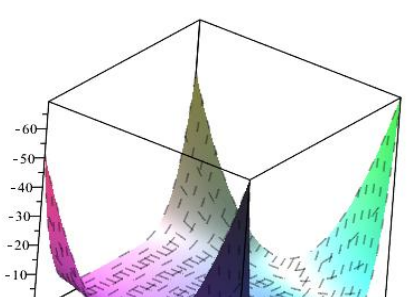


Table (1) computation between the exact and HAM $h=-1$ in formula 2 and determine the absolute error

\begin{tabular}{|c|c|c|c|c|c|c|}
\hline$x$ & exact $\underline{u}(x, r)$ & $\begin{array}{c}\text { HAM } \underline{u}(x, r) \\
\alpha=0.1\end{array}$ & exact $\underline{u}(x, r)$ & $\begin{array}{c}\text { HAM } \underline{u}(x, r) \\
\alpha=0.5\end{array}$ & exact $\underline{u}(x, r)$ & $\begin{array}{c}\text { HAM } \underline{u}(x, r) \\
\alpha=1\end{array}$ \\
\hline 0 & 0.00000 & 0.0000000 & 0.00000 & 0.00000000 & 0.00000 & 0.000000000 \\
\hline 0.2 & 0.04000 & 0.0477480 & 0.02000 & 0.02082064 & 0.04000 & 0.039294998 \\
\hline 0.4 & 0.01600 & 0.0167550 & 0.08000 & 0.07969799 & 0.16000 & 0.1569610105 \\
\hline 0.6 & 0.03600 & 0.0263958 & 0.18000 & 0.17088774 & 0.36000 & 0.3544127825 \\
\hline 0.8 & 0.06400 & 0.0594177 & 0.32000 & 0.26787734 & 0.64000 & 0.5662234183 \\
\hline 1 & 0.10000 & 0.1360818 & 0.50000 & 0.48953632 & 1.00000 & 0.9882424058 \\
\hline
\end{tabular}

\begin{tabular}{|c|c|c|c|c|c|c|}
\hline$x$ & exact $\bar{u}(x, r)$ & $\begin{array}{c}\text { HAM } \bar{u}(x, r) \\
\alpha=0.1\end{array}$ & exact $\bar{u}(x, r)$ & $\begin{array}{c}\text { HAM } \bar{u}(x, r) \\
\alpha=0.5\end{array}$ & exact $\bar{u}(x, r)$ & $\begin{array}{c}\text { HAM } \bar{u}(x, r) \\
\alpha=1\end{array}$ \\
\hline 0 & 0.00000 & 0.000000000 & 0.0000 & 0.0000000000 & 0.0000 & 0.000000000 \\
\hline 0.2 & 0.07600 & 0.068505806 & 0.0600 & 0.0576338330 & 0.0400 & 0.035410480 \\
\hline 0.4 & 0.30400 & 0.280978546 & 0.2400 & 0.2260091416 & 0.1600 & 0.151048729 \\
\hline 0.6 & 0.68400 & 0.655780340 & 0.5400 & 0.5168401528 & 0.3600 & 0.358551838 \\
\hline 0.8 & 1.12160 & 1.161509992 & 0.9600 & 0.8996547609 & 0.6400 & 0.639295323 \\
\hline 1 & 1.90000 & 1.875435586 & 1.5000 & 1.5511618405 & 1.0000 & 0.999216891 \\
\hline
\end{tabular}




\section{Example 2}

Now we discus this example

We will discuss Now the case of formula $2, \underline{k\left(x, t, F_{1 \alpha}(t, u(t, \alpha))\right)} \geq 0, G \overline{\left(t, \int_{a}^{t} F_{2 \alpha}(t, s, u(s, \alpha)) d s\right)} \geq 0$ $0 \leq t \leq \frac{1}{2} \quad$, and $\underline{k\left(x, t, F_{1 \alpha}(t, u(t, \alpha))\right)}<0, G \overline{\left(t, \int_{a}^{t} F_{2 \alpha}(t, s, U(s, \alpha)) d s\right)}<0,1 / 2 \leq t \leq x \quad, \mathrm{c}=1 / 2$ $\underline{u}(x, \alpha)=\underline{f}(x, \alpha)+\lambda \int_{a}^{c} k\left(x, t, \underline{F}_{1 \alpha}(t, \underline{u}(t, \alpha))\right) d t+\lambda \int_{c}^{x} k\left(x, t, G\left(t, \int_{a}^{t} \bar{F}_{2 \alpha}(t, s, \bar{u}(s, \alpha)) d s\right) d t\right.$ $\bar{u}(x, \alpha)=\bar{f}(x, \alpha)+\lambda \int_{a}^{c} k\left(x, t, G\left(t, \int_{a}^{t} \bar{F}_{2 \alpha}(t, s, \bar{u}(s, \alpha)) d s\right) d t+\lambda \int_{c}^{x} k\left(x, t, \underline{F}_{1 \alpha}(t, \underline{u}(t, \alpha))\right) d t\right.$

Where

$$
\begin{aligned}
k\left(x, t, \underline{F}_{1 \alpha}(t, \underline{u}(t, \alpha))\right) & =x t(\underline{u}(x, \alpha))^{2} \\
G\left(t, \int_{a}^{t} \bar{F}_{2 \alpha}(t, s, \bar{u}(s, \alpha)) d s\right) & =x t \cdot \int_{a}^{t}(\bar{u}(s, \alpha))^{2} d s
\end{aligned}
$$

$\begin{aligned} f(x, \alpha)= & \left(\frac{5}{4}\right. \\ & \left.-\frac{1}{4} r\right) \mathrm{e}^{x}-0.05760255714 r^{2}+0.5135255714 r-1.377563928+\frac{1}{64} x^{2} r^{2}+\frac{3}{32} x^{2} \\ & +\frac{9}{64} x^{2}-\frac{1}{64} \mathrm{e}^{2 x} r^{2} x+\frac{1}{128} \mathrm{e}^{2 x} r^{2}-\frac{3}{32} \mathrm{e}^{2 x} r x+\frac{3}{64} \mathrm{e}^{2 x} r-\frac{9}{64} x \mathrm{e}^{2 x} \\ & +\frac{9}{128} \mathrm{e}^{2 x}\end{aligned}$

$$
\begin{aligned}
\bar{f}(x, \alpha)=\left(\frac{3}{4}\right. & \left.+\frac{1}{4} r\right) \mathrm{e}^{x}+2.088501429+0.08104005716 r^{2}-0.8729005716 r-\frac{1}{32} \mathrm{e}^{2 x} r^{2} \\
& +\frac{5}{16} \mathrm{e}^{2 x} r-\frac{25}{32} \mathrm{e}^{2 x}
\end{aligned}
$$

the intimal condition is $\underline{u}_{0}(x, \alpha)=\bar{u}_{0}(x, \alpha)=0$, and $0 \leq \alpha \leq 1$ the exact solution $\underline{u}(x, \alpha)=\left(\frac{5}{4}-\frac{r}{4}\right) \operatorname{ep}(x)$ and $\bar{u}(x, \alpha)=\left(\frac{3}{4}+\frac{r}{4}\right) \exp (x)$

$$
\begin{aligned}
\underline{u}_{1}(x, \alpha)=-h & \left(\left(\frac{5}{4}-\frac{1}{4} r\right) \mathrm{e}^{x}-0.05760255714 r^{2}+0.5135255714 r-1.377563928+\frac{1}{64} x^{2} r^{2}\right. \\
& +\frac{3}{32} x^{2} r+\frac{9}{64} x^{2}-\frac{1}{64} \mathrm{e}^{2 x} r^{2} x+\frac{1}{128} \mathrm{e}^{2 x} r^{2}-\frac{3}{32} \mathrm{e}^{2 x} r x+\frac{3}{64} \mathrm{e}^{2 x} r-\frac{9}{64} x \mathrm{e}^{2 x} \\
& \left.+\frac{9}{128} \mathrm{e}^{2 x}\right)
\end{aligned}
$$




$$
\begin{aligned}
\bar{u}_{1}(x, \alpha)=-h & \left(\left(\frac{3}{4}+\frac{1}{4} r\right) \mathrm{e}^{x}+2.088501429+0.08104005716 r^{2}-0.8729005716 r-\frac{1}{32} \mathrm{e}^{2 x} r^{2}\right. \\
& \left.+\frac{5}{16} \mathrm{e}^{2 x} r-\frac{25}{32} \mathrm{e}^{2 x}\right)
\end{aligned}
$$


$\underline{u}_{2}(x, \alpha)$ 
$=$

$-h\left(\left(\frac{5}{4}-\frac{1}{4} r\right) e^{x}-0.05760255714 r^{2}+0.5135255714 r-1.377563928+\frac{1}{64} x^{2} r^{2}\right.$ $+\frac{3}{32} x^{2} r+\frac{9}{64} x^{2}-\frac{1}{64} \mathrm{e}^{2 x} r^{2} x+\frac{1}{128} \mathrm{e}^{2 x} r^{2}-\frac{3}{32} \mathrm{e}^{2 x} r x+\frac{3}{64} \mathrm{e}^{2 x} r-\frac{9}{64} x \mathrm{e}^{2 x}$ $\left.+\frac{9}{128} e^{2 x}\right)-(1+h) \cdot\left(h \cdot\left(\left(\frac{5}{4}-\frac{r}{4}\right) \cdot \exp (x)-0.05369630714 r^{2}+0.5369630714 r\right.\right.$ $-1.342407678-0.03515625000-0.003906250000 r^{2}-0.02343750000 r+\frac{1}{64} x^{2} r^{2}$ $+\frac{3}{32} x^{2} r+\frac{9}{64} x^{2}-\frac{1}{64} \mathrm{e}^{2 x} r^{2} x+\frac{1}{128} \mathrm{e}^{2 x} r^{2}-\frac{3}{32} \mathrm{e}^{2 x} r x+\frac{3}{64} \mathrm{e}^{2 x} r-\frac{9}{64} x \mathrm{e}^{2 x}$ $\left.\left.+\frac{9}{128} e^{2 x}\right)\right)-h \cdot\left(\left(0.07219843022 h^{4}+0.1443968604 h^{3}+0.001285261232 h^{4} r^{4}\right.\right.$ $-0.01163777838 h^{4} r^{3}+0.002570522464 h^{3} r^{4}+0.01223394163 h^{4} r^{2}$ $-0.02327555675 h^{3} r^{3}+0.001285261232 h^{2} r^{4}+0.05885643660 h^{4} r$

$+0.02446788327 h^{3} r^{2}-0.01163777838 h^{2} r^{3}+0.1177128732 h^{3} r$

$\left.+0.01223394163 h^{2} r^{2}+0.05885643660 h^{2} r+0.07219843022 h^{2}\right)-($

$-0.5804907395 h^{4} r x^{2}-1.160981480 h^{3} r x^{2}+0.001144180580 h^{4} r^{4} x^{2}$

$+0.002288361161 h^{3} r^{4} x^{2}-0.04151602174 h^{4} r^{3} x^{2}-0.08303204345 h^{3} r^{3} x^{2}$

$+0.3059602080 h^{4} r^{2} x^{2}+0.6119204160 h^{3} r^{2} x^{2}-0.5804907395 h^{2} r x^{2}$

$+0.001144180580 h^{2} r^{4} x^{2}-0.04151602174 h^{2} r^{3} x^{2}+0.3059602080 h^{2} r^{2}$

$-1.215369394 h^{4} r x^{3}-2.430738788 h^{3} r x^{3}+0.002189163621 h^{4} r^{4} x^{3}$

$+0.004378327243 h^{3} r^{4} x^{3}-0.04715994147 h^{4} r^{3} x^{3}-0.09431988297 h^{3} r^{3} x^{3}$

$+0.3668199860 h^{4} r^{2} x^{3}+0.7336390723 h^{3} r^{2} x^{3}-1.215369394 h^{2} r x^{3}$

$+0.002189163621 h^{2} r^{4} x^{3}-0.04715994147 h^{2} r^{3} x^{3}+0.3668199860 h^{2} r^{2} x^{3}$

$-0.04052002858 \mathrm{e}^{x} h^{2} r^{3}+0.3148902001 \mathrm{e}^{x} h^{2} r^{2}+0.2651001429 \mathrm{e}^{x} h^{2} r$

$-0.04052002858 e^{x} h^{4} r^{3}-0.08104005716 e^{x} h^{3} r^{3}+0.3148902001 e^{x} h^{4} r^{2}$

$+0.6297804001 e^{x} h^{3} r^{2}+0.2651001429 e^{x} h^{4} r+0.5302002858 e^{x} h^{3} r$

$+3.132752144 e^{x} h^{2} x+3.132752144 e^{x} h^{4} x+6.265504287 e^{x} h^{3} x-0.7721616465 h^{2} x^{2}$

$0.7721616465 h^{4} x^{2}-1.544323292 h^{3} x^{2}+1.453946073 h^{2} x^{3}+1.453946073 h^{4} x^{3}$

$+2.907892146 h^{3} x^{3}+0.04052002858 e^{x} h^{2} r^{3} x-0.3148902001 e^{x} h^{2} r^{2} x$

$-0.2651001429 e^{x} h^{2} r x+0.04052002858 e^{x} h^{4} r^{3} x+0.08104005716 e^{x} h^{3} r^{3} x$

$-0.3148902001 \mathrm{e}^{x} h^{4} r^{2} x-0.6297804001 \mathrm{e}^{x} h^{3} r^{2} x-0.2651001429 \mathrm{e}^{x} h^{4} r x$

$-0.5302002858 \mathrm{e}^{x} h^{3} r x-0.3805275670 h^{2} r \mathrm{e}^{2 \cdot x}-0.00001525878906 h^{2} r^{4} \mathrm{e}^{4 \cdot x}$

$+0.001157407408 h^{3} r^{3} \mathrm{e}^{3 . x}+0.0003051757812 h^{4} r^{3} \mathrm{e}^{4 \cdot x}+0.0006103515625 h^{3} r^{3} \mathrm{e}^{4 . x}$

$+0.0006331254465 h^{4} r^{4} e^{2 \cdot x}-0.002288818359 h^{4} r^{2} e^{4 . x}+0.007629394531 h^{2} r e^{4 . x}$

- $0.002893518519 h^{4} r e^{3 . x}-0.005787037037 h^{3} r e^{3 . x}-0.004577636719 h^{3} r^{2} e^{4 x}$

- 0.01315079018 $h^{4} r^{3} e^{2 \cdot x}-0.02630158035 h^{3} r^{3} e^{2 \cdot x}+0.001266250893 h^{3} r^{4} e^{2 . x}$

$0.004050925926 h^{4} r^{2} e^{3 \cdot x}-0.008101851852 h^{3} r^{2} e^{3 \cdot x}+0.0005787037037 h^{2} r^{3} e^{3 . x}$

$+0.0003051757812 h^{2} r^{3} e^{4 \cdot x}+0.09252741072 h^{2} r^{2} e^{2 \cdot x}-0.3805275670 h^{4} r e^{2 . x}$

$-0.7610551340 h^{3} r \mathrm{e}^{2 \cdot x}-0.00001525878906 h^{4} r^{4} \mathrm{e}^{4 \cdot x}-0.00003051757812 h^{3} r^{4} \mathrm{e}^{4 \cdot x}$

$+0.0005787037037 h^{4} r^{3} \mathrm{e}^{3 . x}+0.007629394531 h^{4} r \mathrm{e}^{4 \cdot x}+0.01525878906 h^{3} r \mathrm{e}^{4 . x}$

$+0.09252741072 h^{4} r^{2} e^{2 \cdot x}+0.1850548215 h^{3} r^{2} e^{2 \cdot x}-0.002893518519 h^{2} r e^{3 . x}$

$+0.0006331254465 h^{2} r^{4} e^{2 \cdot x}-0.004050925926 h^{2} r^{2} e^{3 . x}-0.002288818359 h^{2} r^{2} e^{4 \cdot x}$

- $0.01315079018 h^{2} r^{3} e^{2 \cdot x}-0.1302083333 h^{2} x e^{3 \cdot x}+0.07629394530 h^{3} x e^{4 . x}$

- $0.6751958705 h^{2} x \mathrm{e}^{2 \cdot x}-0.2604166667 h^{3} x \mathrm{e}^{3 \cdot x}+0.03814697265 h^{4} x \mathrm{e}^{4 . x}$

$0.1302083333 h^{4} x \mathrm{e}^{3 \cdot x}+0.03814697265 h^{2} x \mathrm{e}^{4 \cdot x}-0.6751958705 h^{4} x \mathrm{e}^{2 . x}$

$-1.350391742 h^{3} x e^{2 \cdot x}+1.522110268 h^{3} r x e^{2 \cdot x}+0.00006103515625 h^{4} r^{4} x e^{4 . x}$

$+0.0001220703125 h^{3} r^{4} x e^{4 \cdot x}-0.001736111111 h^{4} r^{3} x e^{3 . x}$

- $0.03051757812 h^{4} r x e^{4 \cdot x}-0.06103515625 h^{3} r x e^{4 \cdot x}-0.1850548214 h^{4} r^{2} x e^{2 . x}$

$-0.3701096430 h^{3} r_{x}^{2} e^{2 \cdot x}+0.008680555557 h^{2} r x e^{3 \cdot x}-0.001266250893 h^{2} r^{4} x e^{2 . x}$

$+0.01215277778 h^{2} r^{2} x e^{3 \cdot x}+0.009155273438 h^{2} r^{2} x e^{4 \cdot x}+0.02630158036 h^{2} r^{3} x e^{2 . x}$

$+0.7610551340 h^{2} r x \mathrm{e}^{2 \cdot x}+0.00006103515625 h^{2} r^{4} x \mathrm{e}^{4 \cdot x}$

- $0.003472222223 h^{3} r^{3} x \mathrm{e}^{3 \cdot x}-0.001220703125 h^{4} r^{3} x \mathrm{e}^{4 \cdot x}$

$0.002441406250 h^{3} r^{3} x e^{4 \cdot x}-0.001266250893 h^{4} r^{4} x e^{2 \cdot x}$

$+0.009155273438 h^{4} r^{2} e^{4 \cdot x}-0.03051757812 h^{2} r x e^{4 \cdot x}+0.008680555557 h^{4} r x e^{3 . x}$

$+0.01736111111 h^{3} r x e^{3 \cdot x}+0.01831054688 h^{3} r^{2} x e^{4 \cdot x}+0.02630158036 h^{4} r^{3} x e^{2 \cdot x}$

$+0.05260316070 h^{3} r^{3} x e^{2 \cdot x}-0.002532501786 h^{3} r^{4} x e^{2 \cdot x}+0.01215277778 h^{4} r^{2} x e^{3 . x}$

$+0.02430555556 h^{3} r^{2} x e^{3 . x}-0.001736111111 h^{2} r^{3} x e^{3 . x}-0.001220703125 h^{2} r^{3} x e^{4 .}$

$0.1850548214 h^{2} r^{2} x e^{2 . x}+0.7610551340 h^{4} r x e^{2 x}+2.620606047 h^{4}$

$+5.241212094 h^{3}+0.6751958708 h^{3} \mathrm{e}^{2 \cdot x}+0.04340277778 h^{2} \mathrm{e}^{3 \cdot x}$

- $0.01907348632 h^{3} \mathrm{e}^{4 \cdot x}+0.3375979352 h^{2} \mathrm{e}^{2 \cdot x}+0.08680555556 h^{3} \mathrm{e}^{3 \cdot x}$

- $0.009536743162 h^{4} \mathrm{e}^{4 \cdot x}+0.04340277778 h^{4} \mathrm{e}^{3 \cdot x}-0.009536743162 h^{2} \mathrm{e}^{4 \cdot x}$

$+0.3375979352 h^{4} e^{2 \cdot x}-0.0006724386462 h^{4} r^{4}+0.05322886062 h^{4} r^{3}$

$-0.001344877292 h^{3} r^{4}-0.4079153381 h^{4} r^{2}+0.1064577212 h^{3} r^{3}$

- $0.0006724386462 h^{2} r^{4}+0.1283958359 h^{4} r-0.8158306763 h^{3} r^{2}$

$+0.05322886062 h^{2} r^{3}+0.2567916720 h^{3} r-0.4079153381 h^{2} r^{2}+0.1283958359 h^{2} r$

$\left.-3.132752144 e^{x} h^{2}-3.132752144 e^{x} h^{4}-6.265504287 e^{x} h^{3}+2.620606047 h^{2}\right)$ ) 


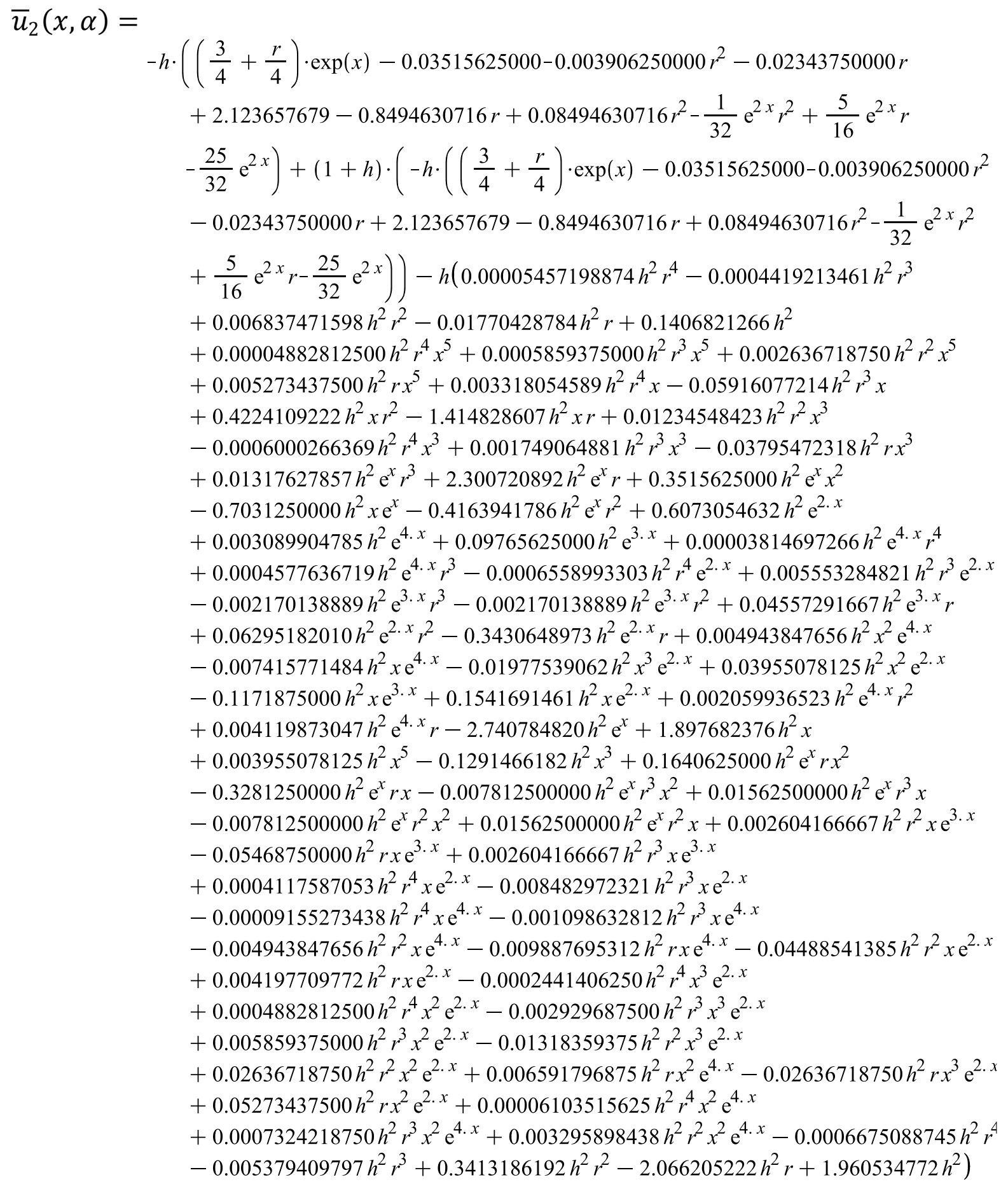


Fig(3a)Exact solution $\underline{u}(x, \alpha) \operatorname{Fig}(3 b)$ Approximate solution $\underline{u}(x, \alpha)$
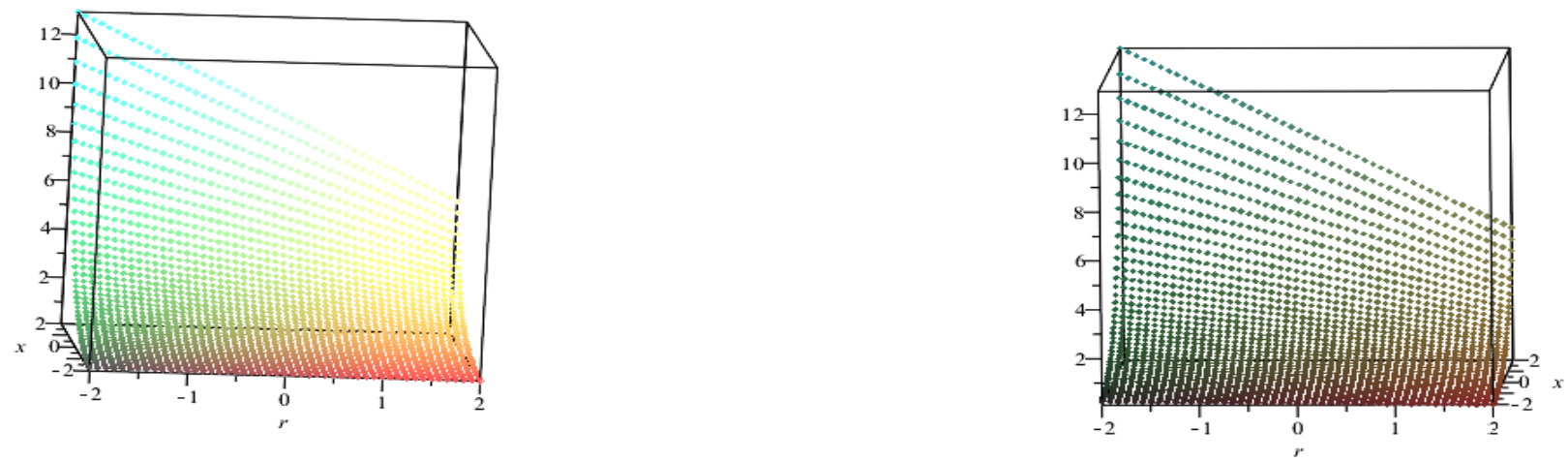

Fig(4a)Exact solution $\bar{u}(x, \alpha) \operatorname{Fig}(4 \mathrm{~b})$ Approximate solution $u(x, \alpha)$
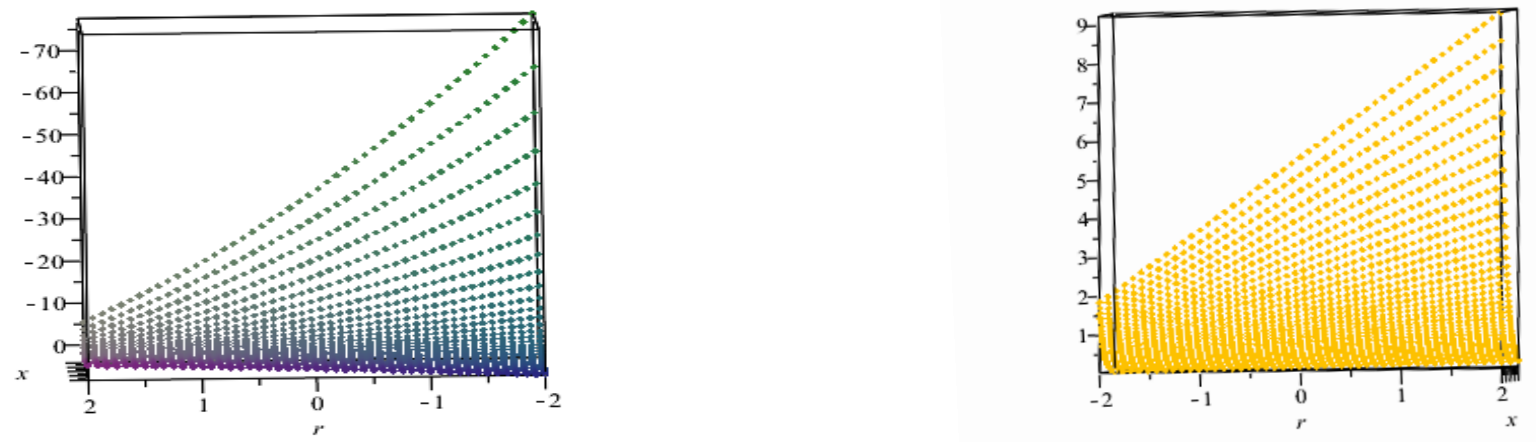

Table (2) computation between the exact and HAM $h=-1$ and determine the absolute error

\begin{tabular}{|c|c|c|c|c|c|c|}
\hline$x$ & exact $\underline{u}(x, r)$ & $\begin{array}{c}\text { HAM } \underline{u}(x, r) \\
\alpha=0.1\end{array}$ & exact $\underline{u}(x, r)$ & $\begin{array}{c}\text { HAM } \underline{u}(x, r) \\
\alpha=0.5\end{array}$ & exact $\underline{u}(x, r)$ & $\begin{array}{c}\text { HAM } \underline{u}(x, r) \\
\alpha=1\end{array}$ \\
\hline 0 & 1.225000 & 1.2250000 & 1.125000 & 1.1250000 & 1.000000 & 1.000000000 \\
\hline 0.2 & 1.496218 & 1.4283208 & 1.374078 & 1.3989047 & 1.221403 & 1.183280129 \\
\hline 0.4 & 1.827485 & 1.7853893 & 1.673028 & 1.5618896 & 1.491825 & 1.465080107 \\
\hline 0.6 & 2.232096 & 1.9869999 & 2.049884 & 1.9880279 & 1.822119 & 1.763887097 \\
\hline 0.8 & 2.726287 & 2.2975165 & 2.503734 & 2.0827974 & 2.225541 & 2.180635898 \\
\hline 1 & 3.329852 & 3.2589450 & 3.058067 & 2.9980588 & 2.718282 & 2.689145141 \\
\hline
\end{tabular}




\begin{tabular}{|c|c|c|c|c|c|c|}
\hline $\mathrm{x}$ & exact $\bar{u}(x, r)$ & $\begin{array}{c}\operatorname{HAM} \bar{u}(\mathrm{x}, \mathrm{r}) \\
\alpha=0.1\end{array}$ & exact $\bar{u}(x, r)$ & $\begin{array}{c}\operatorname{HAM} \bar{u}(\mathrm{x}, \mathrm{r}) \\
\alpha=0.5\end{array}$ & $\operatorname{exactu}(\mathrm{x}, \mathrm{r})$ & $\begin{array}{c}\operatorname{HAM} \bar{u}(\mathrm{x}, \mathrm{r}) \\
\alpha=1\end{array}$ \\
\hline 0 & 0.775000 & 0.775000000 & 0.875000 & 0.87500000 & 1.000000 & 1.00000000 \\
\hline 0.2 & 0.946587 & 1.006239408 & 1.068727 & 1.157998195 & 1.221403 & 1.253657499 \\
\hline 0.4 & 1.156164 & 1.207942563 & 1.305347 & 1.342539991 & 1.491825 & 1.511335517 \\
\hline 0.6 & 1.412142 & 1.394015112 & 1.594354 & 1.489358232 & 1.822119 & 1.785015292 \\
\hline 0.8 & 1.724794 & 1.662404563 & 1.947348 & 1.886852525 & 2.225541 & 2.198045673 \\
\hline 1 & 2.106668 & 1.998260457 & 2.378497 & 2,287056234 & 2.718282 & 2.667938815 \\
\hline
\end{tabular}

\section{CONCLUSION}

The proposed method is a powerful procedure for solving new type fuzzy nonlinear Volterra integral equation. The examples analyzed illustrate the ability and reliability of the method presented in this paper and severals that the one is very simple and effective. The obtained solutions, in comparison with the exact solution admit a remarkable accuracy. Results indicate that the convergence rate is very fast, and lower approximations can achieve high accuracy

\section{References}

[1] Nor hanim abd.rahman,arsmah Ibrahim ,mohd idris jays, newton homotopy solution for nonlinear equations using maple 14 ,faculty of computer and mathematical scienes university teknologi mara malyusia(uitm) 40450 Shah Alam, Selangor ,Malaysia volume .3 , number 2 Decamber(2011)

[2] M.Ghanbari , numerical solution of fuzzy linear volterra integral equation of the second kind by homotopy analysis method, Department of mathematics, science and research branch , Islamic Azad University, Tehran , Iran ,Received 10 january 2010..

[3] Eman A. Hussain, Ayad W. Ali , Homotopy Analysis Method for solving Non linear fuzzy integral equation, Department of Mathematics, College of Science, Al-Mustansiriyah Baghdad, Iraq (2011).

[4] Edyta H. Damian Slota, Tomasz. T, Roman Wituda. Usage of the Homotopy analysis method for solving the Non Linear and linear integral equation of the second kind (2013). Northeland.

[5] R. Goetschel and Vaxman, Elementry fuzzy clculus, Fuzzy Sets and Systems, 18 (1986), 3143

[6] Sarmad A. Altari, Numerical Solutions of Fuzzy Fredholm Integral Equation of the second kind using Bernstein Polynomials, Department of Computer Engineering and Information Technology University of Technology, Baghdad - Iraq (2012)

[7] Hany. N. Magdy. A, A new technique of using Homotopy Analysis Method for Second Order Non Linear Differential Equation, Department of Basic Science, Faculty of Engineering of Branch Benha University, Egypt (2012)

[8] N. A. Rajab, A. M. Ahmad, O. M. Alfaour, Reduction Formula for Linear Fuzzy Equation, Applied Science Department, University of Technology Baghdad - Iraq (2013) 
[9] Sushila Rathora, Devendra Kumar, Jagdev Singh, Sumit Gapta, Homotopy Analysis Method for Non Linear Equation, Department of Mathematics, Jagon Meth, University Village - Rampun Tehsil Chaksu, Jaipur - 303901, Ragashtan - India (2012)

[10] S. Abbasbandy, E. Babolian, M. Alavi, Numerical method for solving linear Fredholm fuzzy integral equations of the second kind, Chaos Soliton and Fractals 31 (2007) 138146.

[11] Abbasbandy, S., Msgyai, E., \& Shivanian, E. (2009). The homotopy analysis method for multiple solutions of nonlinear boundary value problems. Commun Nonlinear Sci. Num. Simulat.,14(9-10), 3530-3536.

[12] H.C. Wu, The improper fuzzy Riemann integral and its numerical integration, Information Science 111 (1999) 109-137.

[13] S. Abbasbandy and A. Jafarian, "Steepest descent method for solving fuzzy nonlinear equations," Applied Mathematics and Computation, vol. 174, no. 1, pp. 669-675, 2006.

[14].H.C.Wu, The proper fuzzy Riemann integral and its numerical integration, Information Science 111(1999) 109-137

[15] G. J. Klir, U. St. Clair, and B. Yuan, Fuzzy Set Theory: Foundations and Applications, Prentice-Hall, Eaglewood Cliffs ,NJ, USA, 1997.

[16] J.Y. Park, Y.C. Kwan, J.V. Jeong, Existence of solutions of fuzzy integral equationsin Banach spaces, Fuzzy Sets and System 72 (1995) 373-378

[17]. Chang, S. S. L.,\& Zadah, L. A. (1972). On fuzzy mapping and control. Trans. Systems, Man Cyberetics, SMC-2(1), 30-34.

[18]. S. Abbasbandy, "The application of homotopy nanlysis mthod to nonlinear equations arising in heat transfer," Phys. Lett. A, vol.360, pp 109-113, 2006

[19].S.J. Liao, Notes on the homotopy analysis method: Some definitions and theorems, Commun Nonlinear Sci. Numer. Simulat. 14 (2009) 983-997.

[20].J.Y.Park, Y.C.Kwan,J.V. Jeong, Existence of solution of fuzzy integral equations in Banach spaces, Fuzzy Sets and System 72 ( 1995) 373- 378.

[21]D. Dubois, H. Prade, Operation on fuzzy numbers, Int. J. system Science 9 (1978) 613-626 http://dx.doi.org/10.1080/00207727808941724 
[22]. Nanda, S.(1989). On integration of fuzzy mappings. Fuzzy Sets and Systems, 32, 95-101. [23]. R. Goetschel and W. Vaxman, Elementary fuzzy calculus, Fuzzy Sets and Systems, 18 (1986), 31-43

[24]. M. Mizumoto, K. Tanaka, The four operations of arithmetic on fuzzy numbers, Systems Comput. Controls 7(1976) 73-81.

[25]. O. Kaleva, Fuzzy differential equations, Fuzzy Sets and Systems 24 (1987) 301-317 http://dx.doi.org/10.1016/0165-0114(87)90029-7. 